\title{
Anticancer Activity of New Substituted Pyrimidines, Their Thioglycosides and Thiazolopyrimidine Derivatives
}

\author{
Mahmoud N. M. Yousif ${ }^{1}$, Wael A. El-Sayed ${ }^{1}{ }^{*}$, Hebat-Allah S. Abbas $^{1,2}$, Hanem M. Awad $^{3}$, Nabil M. Yousif $^{1}$ \\ ${ }^{1}$ Photochemistry Department, National Research Centre, El-Behouth St, Dokki, Cairo, Egypt. \\ ${ }^{2}$ Chemistry Department, College of Science, King Khalid University, Abha, Saudi Arabia. \\ ${ }^{3}$ Tanning Materials and Leather Technology Department, National Research Centre, El-Behouth St, Dokki, Cairo, Egypt.
}

\author{
ARTICLE INFO \\ Article history: \\ Received on: 10/07/2017 \\ Accepted on: 21/09/2017 \\ Available online: $30 / 11 / 2017$ \\ Key words: Pyrimidines, \\ Thiazolopyrimidines, \\ Thioglycosides, Anticancer, \\ HCT-116, PC-3.
}

\begin{abstract}
Novel functionalized pyrimidine, thioxopyrimidine, iminopyrimidine derivatives and their derived bicyclic thiazolopyrimidine compounds were synthesized. The substituted arylidine derivatives of the thiazolopyrimidine compounds were also prepared. Glycosylation of the thiopyrimidine derivative resulted in formation of the acetylated thioglycosides which were deacetylated to the free hydroxythioglycosides. The synthesized compounds were studied for their anticancer activity against hepatocellular carcinoma HepG-2, human prostate adenocarcinoma PC-3 and human colorectal carcinoma HCT-116 cell lines. Compounds 7c, 8a and 12a showed high activity against PC-3 cancer cells while compounds $\mathbf{1 1 b}$ and 12a revealed higher activity against HCT-116 cell line.
\end{abstract}

\section{INTRODUCTION}

The chemistry and biological research on pyrimidines and their derivatives have attracted great attention because such ring system represents the main skeleton in alkaloids and nucleic bases in addition to their interesting potent biological activities. The anticancer (Abdel Mohsen et al., 2010; El-Sayed et al. 2009), antiviral (El-Sayed et al., 2009; 2008), antibacterial (ElSayed and Abdel-Rahman, 2010; Ramez et al., 2010) antifungal (Gholap et al., 2008), anti-inflammatory (Da et al., 2006) and central nervous activities (Gillespie et al., 2009) properties of many pyrimidine derivatives are well reported. New 4,6diarylpyrimidine compounds have been found to exhibit antitubercular, antibacterial and antiviral activity (Siddiqui et al., 2007). Pyrimidinedione derivatives have been reported to possess antibacterial and anticancer activities (Haiba et al., 2013; Singh

\footnotetext{
* Corresponding Author

Email: waelshendy@gmail.com (Wael A.El-Sayed)
}

and Paul, 2006). Thiazolopyrimidenes represent an important class of fused pyrimidine compounds due to their inhibition activity of 2-methylerythritol-2,4-cyclodiphosphate synthase (Geist et al., 2010). Such type of compounds have been shown to hav antiparkinsonian, analgesic (Amr et al., 2008), anticancer (Flefel et al., 2007; Said et al., 2004) and antimicrobial (Rashad et al., 2010) agents, in addition to activity as phosphate (Kolb et al., 2009) and acetylcholinesterase inhibitors (Zhi et al., 2008). Aromatic thiophenes have attracted much attention as target molecules due to the wide spectrum of its biological activities. These compounds exhibit potent antitumor (Brault et al., 2005) and antibacterial effects (Alagarsamy et al., 2006). On the other hand, extensive interest has been gained to the chemistry and biological properties of glycosyl heterocycles incorporating modified sugar and/or and heterocyclic constituents as biological inhibitors (El Ashry et al., 2007; El-Sayed et al., 2016; 2017). Interestingly, the possible potential hydrogen bond donors and acceptors as a result of presence of polyhydroxyl chain in thioglycosides are expected to promote their affinity toward protein and nucleic acid (Kumar et al., 2015). 
<smiles>S=c1nc(Br)cc(Br)[nH]1</smiles>
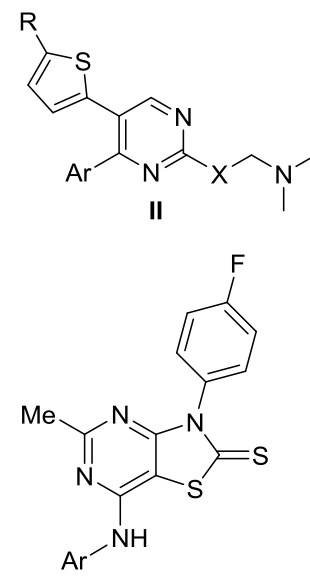

VI

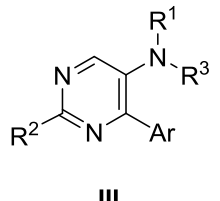

III

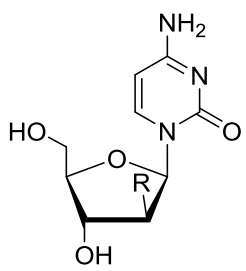

IV; Cladribine or Clofarabine<smiles>Cc1nc2c(sc(=S)n2-c2ccc(F)cc2)c(=O)n1C=N[Ga]</smiles>

Fig. 1: Anticancer Disubstituted pyrimidines, thiazolopyrimidines and pyrimidine sugar Derivatives

Many pyrimidine nucleosides and analogues have been synthesized and studied for anticancer behaviour, and two are in general clinical use. A number of nucleosides and 5-fluorouracil were applied orderly for remediation of breast cancer, gastrointestinal tract tumours in addition to other solid tumours (MacCoss and Robins, 1990). Nucleosides bearing pyranosyl rings have been evaluated for their potential antiviral (Ostrowski et al. 1998; Maurinsh et al., 1997), antioxidant (Spanou et al., 2007) and antibiotic (Haouz et al., 2003) properties and as building blocks in nucleic acid synthesis (Vastmans et al., 2001).

Furthermore, a number of pyrimidine thioglycosides have been shown to have antischistosomal activity (Srour et al., 2009). Compounds I-VI (Abdel Mohsen et al., 2010; Chou et al., 1999; Alagarsamy et al., 2013; Jordheim et al., 2013; Fahmy et al., 2003) (fig. 1) represents examples of reported disubstituted pyrimidine, thiazolopyrimidine and pyrimidinyl sugar derivatives with their anticancer activity. In the same direction and following our program aiming for the synthesis of glycosylthio five and six membered heterocycles, with anticancer, antiviral and antimicrobial activity properties (El-Sayed et al., 2008; 2017; Flefel et al., 2017) we report the synthesis of novel disubstituted pyrimidines, their bicyclic derivatives and their derived thioglycosides with cytotoxic activity evaluation.

\section{MATERIALS AND METHODS}

\section{Instruments and reagents}

All melting points were measured by Electro-thermal IA 9100 apparatus (Shimadzu, Tokyo, Japan) and are uncorrected. Infra-red spectra were investigated $(\mathrm{KBr})$ by means of a PerkinElmer 1650 spectrophotometer (Norwalk, CT, USA). Nuclear Magnetic Resonance $\left({ }^{1} \mathrm{H}\right.$ and $\left.{ }^{13} \mathrm{C} \mathrm{NMR}\right)$ of prepared compounds was carried out on a Jeol-Ex-400 NMR spectrometer (Jeol, Tokyo, Japan) at $25{ }^{\circ} \mathrm{C}$ and chemical shifts were expressed as part per million; ppm ( $\delta$ values) with respect to TMS as internal standard.
Mass spectrometry was performed using VG 2AM-3F spectrometer (Thermo electron corporation, USA). Microanalyses were determined using Mario El Mentar apparatus. Following up the reactions and checking the purity of the compounds were performed by means of TLC which was implemented by aluminum plates pre-coated with silica gel 60 or 60 F254 (Merck) and visualized using UV light $(254 \mathrm{~nm})$.

All chemicals which have been used were of reagent grade and used as provided directly unless otherwise stated. Synthesis of compounds 1a-c and $\mathbf{2 a}$ was performed according to reported procedure (Ramesh and Rao, 2010; Greiner-Bechert and Otto, 1991; Qiang et al., 2013).

\section{General procedure for preparation of compounds $\mathbf{2 b , c}$}

To a solution of enone derivatives $\mathbf{1 b}, \mathbf{c}(10 \mathrm{mmole})$ in dry acetone $(40 \mathrm{~mL}) /$ methanol $(15 \mathrm{~mL})$ mixture, $\mathrm{H}_{2} \mathrm{O}_{2}(20 \%, 15$ $\mathrm{mL})$ and anhydrous $\mathrm{NaOH}(2 \mathrm{~g})$ were added portion-wise. The resulting mixture was stirred in an ice bath until the yellow color is disappeared, then, the temperature was allowed to raise to 30-40 ${ }^{\circ} \mathrm{C}$ for 45 minutes until white clear solution was achieved, then stirring was continued at room temperature for $3 \mathrm{~h}$. Crushed icewater mixture was added and the resulting solid substance was filtered and re-crystallized from ethanol affording the oxirane $\mathbf{2 b , c}$.

\section{(3-(3,4-Dimethoxyphenyl)oxiran-2-yl)(thiophen-2-yl)methanone}

Yield: $61 \%$, m.p. $123-125{ }^{\circ} \mathrm{C}$, yellow powder. IR (KBr) v, $\mathrm{cm}^{-1}: 1697(\mathrm{C}=\mathrm{O}) .{ }^{1} \mathrm{H} \mathrm{NMR}\left(400 \mathrm{MHz}, \mathrm{CDCl}_{3}\right): \delta_{\mathrm{H}} 3.75,3.77$ $\left(2 \mathrm{~s}, 6 \mathrm{H}, 2 \mathrm{OCH}_{3}\right), 3.79(\mathrm{~d}, 1 \mathrm{H}, J=7.2 \mathrm{~Hz}$, oxirane $\mathrm{H}-2), 3.92$ (d, $1 \mathrm{H}, J=7.2 \mathrm{~Hz}$, oxirane H-3), 6.80-6.84 (m, 2H, Ar-H), 7.26 (d, $1 \mathrm{H}, J=7.2 \mathrm{~Hz}, \mathrm{Ar}-\mathrm{H}), 7.52-7.55(\mathrm{~m}, 1 \mathrm{H}$, thiophene-H), $7.68(\mathrm{~d}$, $1 \mathrm{H}, J=7.5 \mathrm{~Hz}$, thiophene-H), $7.92(\mathrm{~d}, 1 \mathrm{H}, J=7.8 \mathrm{~Hz}$, thiophene$\mathrm{H})$. Anal. calcd. for $\mathrm{C}_{15} \mathrm{H}_{14} \mathrm{O}_{4} \mathrm{~S}$ (290.33): C, 62.05; H, 4.86. Found: C, 62.25; H, 4.94. 
Thiophen-2-yl(3-(3,4,5-trimethoxyphenyl)oxiran-2-yl)methanone

(2c)

Yield $73 \%$, m.p. $153-155^{\circ} \mathrm{C}$, yellow powder. IR $(\mathrm{KBr}) \mathrm{v}$, $\mathrm{cm}^{-1}: 1710(\mathrm{C}=\mathrm{O}) .{ }^{1} \mathrm{H}$ NMR $\left(400 \mathrm{MHz}, \mathrm{CDCl}_{3}\right): \delta_{\mathrm{H}} 3.83(\mathrm{~s}, 3 \mathrm{H}$, $\left.\mathrm{OCH}_{3}\right), 3.85\left(\mathrm{~s}, 6 \mathrm{H}, 2 \mathrm{OCH}_{3}\right), 3.90(\mathrm{~d}, 1 \mathrm{H}, J=7.2 \mathrm{~Hz}$, oxirane $\mathrm{H}$ 2), 4.02 (d, 1H, J=7.2 Hz, oxirane H-3), 7.18 (s, 2H, Ar-H), 7.26$7.28(\mathrm{~m}, 1 \mathrm{H}$, thiophene-H), $7.74(\mathrm{~d}, 1 \mathrm{H}, J=7.5 \mathrm{~Hz}$, thiophene-H), $8.00\left(\mathrm{~d}, 1 \mathrm{H}, J=7.5 \mathrm{~Hz}\right.$, thiophene-H). ${ }^{13} \mathrm{C} \mathrm{NMR}(75 \mathrm{MHz}$, $\left.\mathrm{CDCl}_{3}\right): \delta_{\mathrm{c}} 56.2\left(2 \mathrm{CH}_{3} \mathrm{O}\right), 59.6\left(\mathrm{CH}_{3} \mathrm{O}\right), 60.9$ (oxirane-C), 68.9 (oxirane-C), 109.5, 110.7, 120.9, 128.2, 128.5, 130.8, 131.7, 133.6 (Ar-C and thiophene-C), $186.3(\mathrm{C}=\mathrm{O}) . \mathrm{MS}, \mathrm{m} / z(\%)(320.35,2.49$ $\%, \mathrm{M}^{+}$). Anal. calcd. for $\mathrm{C}_{16} \mathrm{H}_{16} \mathrm{O}_{5} \mathrm{~S}$ (320.36): C, 59.99; H, 5.03. Found: C, 59.77; H, 4.86.

\section{General method for preparation of dihydropyrimidines 3a-c}

Thiourea (10 mmole) was added to a stirred mixture of chalcone derivatives 1a-c (10 mmole) and anhydrous sodium hydroxide $(1.5 \mathrm{~g})$ in absolute ethanol $(10 \mathrm{~mL})$ then the reaction mixture was heated under reflux till completion of reaction (TLC; Pet ether/ethyl acetate: 3:1). The resulting mixture was poured onto hydrochloric acid solution $(5 \%, 15 \mathrm{~mL})$ in an ice bath resulting in a yellowish precipitate which was filtered and recrystallized to afford substituted dihydropyrimidine 3a-c, respectively.

\section{4-Phenyl-6-(thiophen-2-yl)-3,4-dihydropyrimidine-2(1H)-thione} (3a)

Yield 93\%, m.p. $160-162{ }^{\circ} \mathrm{C}$, reaction time; 1.5 hour, solvent of crystallization: ethanol/water $(2: 1)$, yellow powder. IR $(\mathrm{KBr}) v, \mathrm{~cm}^{-1}: 3250(\mathrm{NH}) .{ }^{1} \mathrm{H}$ NMR (400 MHz, DMSO-d 6 ): $\delta_{\mathrm{H}}$ $4.57(\mathrm{~d}, 1 \mathrm{H}, J=6.2 \mathrm{~Hz}$, pyrimidine $\mathrm{H}-4), 6.18(\mathrm{~d}, 1 \mathrm{H}, J=6.2 \mathrm{~Hz}$, pyrimidine $\mathrm{H}-5), 6.58-6.64(\mathrm{~m}, 2 \mathrm{H}, \mathrm{Ar}-\mathrm{H}), 6.73(\mathrm{t}, 1 \mathrm{H}, J=7.5 \mathrm{~Hz}$, Ar-H), 6.95-7.01 (m, 3H, Ar-H and thiophene-H), 7.32 $(\mathrm{d}, 1 \mathrm{H}, J=7.5 \mathrm{~Hz}$, thiophene-H), $7.56(\mathrm{~d}, 1 \mathrm{H}, J=7.5 \mathrm{~Hz}$, thiophene-H), 8.50 (brs, $2 \mathrm{H}, \mathrm{NH}) .{ }^{13} \mathrm{C}$ NMR (75 MHz, DMSO-d 6 ): $\delta_{\mathrm{c}} 59.9$ (pyrimidine C-4), 102.5 (pyrimidine C-5), 120.9, 128.3, 128.6, 130.8, 132.5, 135.2, 137.8, 148.6 (Ar-C, thiophene-C and pyrimidine C-6), $176.2(\mathrm{C}=\mathrm{S})$. Anal. calcd. for $\mathrm{C}_{14} \mathrm{H}_{12} \mathrm{~N}_{2} \mathrm{~S}_{2}$ (272.38): C, 61.73; H, 4.44; N, 10.28. Found: C, 61.95; H, 4.58; N, 10.09 .

\section{4-(3,4-Dimethoxyphenyl)-6-(thiophen-2-yl)-3,4- dihydropyrimidine-2(1H)-thione $(3 \mathrm{~b})$}

Yield $87 \%$, m.p. $85-87^{\circ} \mathrm{C}$, reaction time; 1 hour, solvent of crystallization: ethanol, yellow needles. IR $(\mathrm{KBr}) \mathrm{v}, \mathrm{cm}^{-1}: 3310$ $(\mathrm{NH}) .{ }^{1} \mathrm{H}$ NMR $\left(400 \mathrm{MHz}, \mathrm{DMSO}-\mathrm{d}_{6}\right): \delta_{\mathrm{H}} 3.75,3.77(2 \mathrm{~s}, 6 \mathrm{H}$, $\left.2 \mathrm{OCH}_{3}\right), 4.37$ (d, $1 \mathrm{H}, J=6.2 \mathrm{~Hz}$, pyrimidine $\left.\mathrm{H}-4\right), 6.18(\mathrm{~d}, 1 \mathrm{H}, J=$ $6.2 \mathrm{~Hz}$, pyrimidine H-5), 6.53 (d, $1 \mathrm{H}, J=7.5 \mathrm{~Hz}, \mathrm{Ar}-\mathrm{H}), 6.65-6.72$ $(\mathrm{m}, 2 \mathrm{H}, \mathrm{Ar}-\mathrm{H}), 6.95-6.99(\mathrm{~m}, 1 \mathrm{H}$, thiophene-H), $7.21(\mathrm{~d}, 1 \mathrm{H}, J=$ $7.5 \mathrm{~Hz}$, thiophene-H), $7.42(\mathrm{~d}, 1 \mathrm{H}, J=7.5 \mathrm{~Hz}$, thiophene-H), 9.81 (brs, $1 \mathrm{H}, \mathrm{NH}$ ), 11.15 (brs, $1 \mathrm{H}, \mathrm{NH}$ ). Anal. calcd. for $\mathrm{C}_{16} \mathrm{H}_{16} \mathrm{~N}_{2} \mathrm{O}_{2} \mathrm{~S}_{2}$
(332.44): C, 57.81; H, 4.85; N, 8.43. Found: C, 57.55; H, 4.73; N, 8.58 .

\section{6-(Thiophen-2-yl)-4-(3,4,5-trimethoxyphenyl)-3,4- dihydropyrimidine-2(1H)-thione $(3 \mathrm{c})$}

Yield: $73 \%$, m.p. $260-262{ }^{\circ} \mathrm{C}$, reaction time; 1 hour, solvent of crystallization: ethanol, yellow needles. IR $(\mathrm{KBr}) \mathrm{v}, \mathrm{cm}^{-}$ ${ }^{1}$ : $3380(\mathrm{NH}) .{ }^{1} \mathrm{H}$ NMR (400 MHz, DMSO-d $\left.{ }_{6}\right): \delta_{\mathrm{H}} 3.71(\mathrm{~s}, 3 \mathrm{H}$, $\left.\mathrm{OCH}_{3}\right), 3.73\left(\mathrm{~s}, 6 \mathrm{H}, 2 \mathrm{OCH}_{3}\right), 4.01(\mathrm{~d}, 1 \mathrm{H}, J=6.2 \mathrm{~Hz}$, pyrimidine $\mathrm{H}-4), 6.34$ (d, $1 \mathrm{H}, J=6.2 \mathrm{~Hz}$, pyrimidine H-5), 6.78 (s, 2H, Ar-H), 6.86-6.89 (m, 1H, thiophene-H), $6.99(\mathrm{~d}, 1 \mathrm{H}, J=7.5 \mathrm{~Hz}$, thiophene-H), 7.15 (d, 1H, $J=7.5 \mathrm{~Hz}$, thiophene-H), 9.78 (brs, $1 \mathrm{H}, \mathrm{NH}), 11.25$ (brs, $1 \mathrm{H}, \mathrm{NH}) . \mathrm{MS}, m / z(\%)\left(362,1.49 \%, \mathrm{M}^{+}\right)$. Anal. calcd. for $\mathrm{C}_{17} \mathrm{H}_{18} \mathrm{~N}_{2} \mathrm{O}_{3} \mathrm{~S}_{2}$ (362.46): C, 56.33; H, 5.01; N, 7.73. Found: C, 56.18; H, 4.85; N, 7.90.

\section{6-(Thiophen-2-yl)-4-(3,4,5-trimethoxyphenyl)-3,4- dihydropyrimidin-2(1H)-imine (4c)}

A suspension of compound 1c (10 mmole), guanidine hydrochloride (10 mmole) and anhydrous $\mathrm{KOH}(1.5 \mathrm{~g})$ in ethanolwater mixture $(1: 1 ; 15 \mathrm{~mL})$ was refluxed for $5 \mathrm{~h}$. The obtained residue after complete evaporation the solvent was acidified dropwise with cold $\mathrm{HCl}(12 \mathrm{~mL})$ resulting in formation of a yellow solid which was filtered and re-crystallized from ethanol. Yield $61 \%$, m.p. $250-252{ }^{\circ} \mathrm{C}$, yellow powder. IR (KBr) v, $\mathrm{cm}^{-1}: 3260-$ $3240(\mathrm{NH}) .{ }^{1} \mathrm{H}$ NMR (400 MHz, DMSO-d $\left.{ }_{6}\right): \delta_{\mathrm{H}} 3.60(\mathrm{~s}, 3 \mathrm{H}$, $\left.\mathrm{OCH}_{3}\right), 4.64\left(\mathrm{~s}, 6 \mathrm{H}, 2 \mathrm{OCH}_{3}\right), 5.23(\mathrm{~d}, 1 \mathrm{H}, J=6.2 \mathrm{~Hz}$, pyrimidine $\mathrm{H}-4), 5.87$ (d, $1 \mathrm{H}, J=6.2 \mathrm{~Hz}$, pyrimidine $\mathrm{H}-5), 6.84$ (s, 2H, Ar-H), 7.20-7.23 (m, 1H, thiophene-H), $7.34(\mathrm{~d}, 1 \mathrm{H}, J=7.5 \mathrm{~Hz}$, thiophene-H), 7.55 (d, $1 \mathrm{H}, J=7.5 \mathrm{~Hz}$, thiophene-H), 7.94 (brs, $2 \mathrm{H}, 2 \mathrm{NH}$ ), 10.80 (brs, $1 \mathrm{H}, \mathrm{NH}) .{ }^{13} \mathrm{C} \mathrm{NMR}$ (75 MHz, DMSO-d 6 ): $\delta_{\mathrm{c}} 52.9$ (pyrimidine $\left.\mathrm{C}-4\right), 55.8\left(2 \mathrm{OCH}_{3}\right), 55.8\left(\mathrm{OCH}_{3}\right), 102.7$ (pyrimidine C-5), 121.2, 128.4, 128.8, 131.2, 132.6, 135.2, 137.9, 149.3 (Ar-C, thiophene-C and pyrimidine C-6), $155.1 \quad(C=\mathrm{NH})$. Anal. calcd. for $\mathrm{C}_{17} \mathrm{H}_{19} \mathrm{~N}_{3} \mathrm{O}_{3} \mathrm{~S}$ (345.42): C, 59.11; H, 5.54; N, 12.17. Found: 58.96; H, 5.68; N, 12.04.

\section{General procedure for preparation of compounds $5 \mathrm{~b}, \mathrm{c}$}

A mixture of compound $\mathbf{3 b , c}$ (10 mmole), chloroacetic acid (15 mmole), anhydrous sodium acetate $(2 \mathrm{~g})$, acetic anhydride $(8 \mathrm{~mL})$ and glacial acetic acid $(16 \mathrm{~mL})$ was heated at reflux temperature for 2-3 hours. The mixture was poured onto water to afford a brownish solid which was filtered and re-crystallized from acetic acid/water mixture (1:1).

\section{5-(3,4-Dimethoxyphenyl)-7-(thiophen-2-yl)-5H-thiazolo[3,2- a]pyrimidin-3(2H)-one (5b)}

Yield $67 \%$, m.p. $85-87{ }^{\circ} \mathrm{C}$, reaction time; 3 hours, pale brown needles. IR (KBr) v, $\mathrm{cm}^{-1}$ : $1660(\mathrm{C}=\mathrm{O}) .{ }^{1} \mathrm{H}$ NMR for compound $5 \mathbf{b}\left(400 \mathrm{MHz}, \mathrm{DMSO}-\mathrm{d}_{6}\right): \delta_{\mathrm{H}} 3.64\left(\mathrm{~s}, 3 \mathrm{H}, \mathrm{OCH}_{3}\right), 3.68$ $\left(\mathrm{s}, 3 \mathrm{H}, \mathrm{OCH}_{3}\right), 3.78\left(\mathrm{~s}, 2 \mathrm{H}, \mathrm{CH}_{2}\right), 5.33(\mathrm{~d}, 1 \mathrm{H}, J=6.2 \mathrm{~Hz}$, pyrimidine $\mathrm{H}-4), 5.87(\mathrm{~d}, 1 \mathrm{H}, J=6.2 \mathrm{~Hz}$, pyrimidine $\mathrm{H}-5), 6.78$ 6.84 (m, 2H, Ar-H), 7.21 (d, 1H, $J=7.5 \mathrm{~Hz}$, Ar-H), 7.32-7.35 (m, 
1H, thiophene-H), 7.50 (d, $1 \mathrm{H}, J=7.8 \mathrm{~Hz}$, thiophene-H), 7.55 (d, $1 \mathrm{H}, J=7.6 \mathrm{~Hz}$, thiophene-H). Anal. calcd. for $\mathrm{C}_{18} \mathrm{H}_{16} \mathrm{~N}_{2} \mathrm{O}_{3} \mathrm{~S}_{2}$ (372.46): C, 58.05; H, 4.33; N, 7.52. Found: C, 57.88; H, 4.19; N, 7.66 .

7-(Thiophen-2-yl)-5-(3,4,5-trimethoxyphenyl)-5H-thiazolo[3,2a]pyrimidin-3(2H)-one (5c)

Yield $91 \%$, m.p. $85-87^{\circ} \mathrm{C}$, reaction time; 2 hours, yellow needles. IR (KBr) v, $\mathrm{cm}^{-1}: 1670(\mathrm{C}=\mathrm{O}) .{ }^{1} \mathrm{H}$ NMR $(400 \mathrm{MHz}$, DMSO-d $\left.{ }_{6}\right): \delta_{\mathrm{H}} 3.64\left(\mathrm{~s}, 3 \mathrm{H}, \mathrm{OCH}_{3}\right), 3.74\left(\mathrm{~s}, 6 \mathrm{H}, 2 \mathrm{OCH}_{3}\right), 3.80(\mathrm{~s}$, $\left.2 \mathrm{H}, \mathrm{CH}_{2}\right), 3.93(\mathrm{~d}, 1 \mathrm{H}$, pyrimidine $\mathrm{H}-4), 6.64(\mathrm{~d}, 1 \mathrm{H}$, pyrimidine $\mathrm{H}-5), 7.05$ (s, 2H, Ar-H), 7.12-7.15 (m, 1H, thiophene-H), 7.59$7.64\left(\mathrm{~m}, 2 \mathrm{H}\right.$, thiophene-H). ${ }^{13} \mathrm{C} \mathrm{NMR}\left(75 \mathrm{MHz}, \mathrm{DMSO}-\mathrm{d}_{6}\right): \delta_{\mathrm{c}}$ $32.8\left(\mathrm{CH}_{2}\right), 52.9(\mathrm{C}-5), 56.01\left(2 \mathrm{CH}_{3} \mathrm{O}\right), 60.02\left(\mathrm{CH}_{3} \mathrm{O}\right), 107.98(\mathrm{C}-$ 6), 123.8, 124.02, 125.7, 127.5, 135.5, 138.8, 132.2, 138.8, 144.3, 152.8 (Ar-C, thiophene-C, $\mathrm{C}-7$ and $\mathrm{C}=\mathrm{N}), 179.4(\mathrm{C}=\mathrm{O}) . \mathrm{MS}, \mathrm{m} / \mathrm{z}$ (\%) $\left(402,3.19 \%, \mathrm{M}^{+}\right)$. Anal. calcd. for $\mathrm{C}_{19} \mathrm{H}_{18} \mathrm{~N}_{2} \mathrm{O}_{4} \mathrm{~S}_{2}$ (402.48): $\mathrm{C}$, 56.70; H, 4.51; N, 6.96. Found: C, 56.90; H, 4.62; N, 6.79.

\section{General procedure for preparation of compounds $7 \mathrm{~b}, \mathrm{c}$}

A stirred mixture of thiazolopyrimidine derivative $\mathbf{5 b , c}$ (10 mmole), the aldehyde (3,4-dimethoxybenzaldehyde or 3,4,5trimethoxybenzaldehyde) (6a,b) $(10 \mathrm{mmole})$ in absolute ethanol $(20 \mathrm{~mL})$ was heated at reflux temperature for 2-3 hours. Crushed ice-water was then provided affording a precipitate that was filtered and re-crystallized from acetic acid/water (1:1).

\section{2-(3,4-Dimethoxybenzylidene)-5-(3,4-dimethoxyphenyl)-7- (thiophen-2-yl)-5H-thiazolo[3,2-a]pyrimidin-3(2H)-one (7b)}

Yield $78 \%$, m.p. $130-132{ }^{\circ} \mathrm{C}$, reaction time; 2 hours, black powder. IR (KBr) v, $\mathrm{cm}^{-1}$ : $1660(\mathrm{C}=\mathrm{O}) .{ }^{1} \mathrm{H}$ NMR (400 MHz, DMSO-d $\left.{ }_{6}\right): \delta_{\mathrm{H}} 3.59\left(\mathrm{~s}, 3 \mathrm{H}, \mathrm{OCH}_{3}\right), 3.71\left(\mathrm{~s}, 3 \mathrm{H}, \mathrm{OCH}_{3}\right), 3.73(\mathrm{~s}$, $\left.6 \mathrm{H}, 2 \mathrm{OCH}_{3}\right), 5.93(\mathrm{~d}, 1 \mathrm{H}, J=6.2 \mathrm{~Hz}$, pyrimidine $\mathrm{H}-4), 6.03(\mathrm{~d}, J=$ $6.2 \mathrm{~Hz}, 1 \mathrm{H}$, pyrimidine H-5), 7.07-7.14 (m, 3H, Ar-H), 7.18-7.26 (m, 5H, Ar-H and thiophene $\mathrm{H}-3,4), 7.55$ (s, 1H, CH=C), 7.61 (d, $1 \mathrm{H}, J=7.6 \mathrm{~Hz}$, thiophene $\mathrm{H}-5)$. Anal. calcd. for $\mathrm{C}_{27} \mathrm{H}_{24} \mathrm{~N}_{2} \mathrm{O}_{5} \mathrm{~S}_{2}$ (520.62): C, 62.29; H, 4.65; N, 5.38. Found: C, 62.03; H, 4.50; N, 5.51 .

\section{7-(Thiophen-2-yl)-2-(3,4,5-trimethoxybenzylidene)-5-(3,4,5-} trimethoxyphenyl)-5H-thiazolo[3,2-a]pyrimidin-3(2H)-one (7c)

Yield $71 \%$, m.p. $120-122{ }^{\circ} \mathrm{C}$, reaction time; 3 hours, yellow needles. IR (KBr) v, cm-1: $1657(\mathrm{C}=\mathrm{O}) .{ }^{1} \mathrm{H}$ NMR (400 $\left.\mathrm{MHz}, \mathrm{DMSO}-\mathrm{d}_{6}\right): \delta_{\mathrm{H}} 3.63\left(\mathrm{~s}, 6 \mathrm{H}, 2 \mathrm{CH}_{3} \mathrm{O}\right), 3.66\left(\mathrm{~s}, 6 \mathrm{H}, 2 \mathrm{CH}_{3} \mathrm{O}\right)$, $3.70\left(\mathrm{~s}, 6 \mathrm{H}, 2 \mathrm{CH}_{3} \mathrm{O}\right), 5.44(\mathrm{~d}, J=6.2,1 \mathrm{H}$, pyrimidine $\mathrm{H}-4), 6.13$ (d, $1 \mathrm{H}, J=6.2 \mathrm{~Hz}$, pyrimidine H-5), 7.08-7.14 (m, 4H, Ar-H), 7.17-7.21 (m, 2H, thiophene-H), 7.38 (s, 1H, CH=C), 7.45 (d, 1H, $J=7.5 \mathrm{~Hz}$, thiophene-H). Anal. calcd. for $\mathrm{C}_{29} \mathrm{H}_{28} \mathrm{~N}_{2} \mathrm{O}_{7} \mathrm{~S}_{2}$ (580.67): C, 59.99; H, 4.86; N, 4.82. Found: C, 60.28; H, 4.94; N, 4.70.

\section{General method for preparation of compounds 8a-c}

A mixture of the substituted epoxides 2a-c (10 mmole), thiourea $(10 \mathrm{mmole})$ and dry $\mathrm{KOH}(0.5 \mathrm{~g})$ in ethyl alcohol $(10 \mathrm{~mL})$ was refluxed for 45 minutes. The reaction mixture was added portion-wise in a conical flask containing cold hydrochloric acid $(20 \mathrm{~mL})$, filtered, dried and re-crystallized from ethanol/water mixture (1:1).

4-Phenyl-6-(thiophen-2-yl)-2-thioxotetrahydropyrimidin-5(6H)one $(8 a)$

Yield $61 \%$, m.p. $165-167{ }^{\circ} \mathrm{C}$, yellow needles. IR $(\mathrm{KBr}) v$, $\mathrm{cm}^{-1}: 3280(\mathrm{NH}), 1710(\mathrm{C}=\mathrm{O}) .{ }^{1} \mathrm{H}$ NMR (400 MHz, DMSO-d $\left.)_{6}\right): \delta_{\mathrm{H}}$ $3.84(\mathrm{~d}, 1 \mathrm{H}, J=7.2 \mathrm{~Hz}$, pyrimidine-H), $3.86(\mathrm{~d}, 1 \mathrm{H}, J=7.2 \mathrm{~Hz}$, pyrimidine-H), 7.25-7.32 (m, 4H, Ar-H), 7.39-7.52 (m, 4H, Ar-H and thiophene $\mathrm{H}-3,4,5), 9.88$ (brs, $1 \mathrm{H} \mathrm{NH}$ ), 10.27 (brs, $1 \mathrm{H} \mathrm{NH}$ ). Anal. calcd. for $\mathrm{C}_{14} \mathrm{H}_{12} \mathrm{~N}_{2} \mathrm{OS}_{2}$ (288.38): C, 58.31; H, 4.19; N, 9.71. Found: C, 58.14; H, 4.05; N, 9.86 .

\section{4-(3,4-Dimethoxyphenyl)-6-(thiophen-2-yl)-2- \\ thioxotetrahydropyrimidin-5(6H)-one $(8 \mathrm{~b})$}

Yield $73 \%$, m.p. $95-97{ }^{\circ} \mathrm{C}$, yellow needles. IR (KBr) v, $\mathrm{cm}^{-1}: 3310(\mathrm{NH}), 1706(\mathrm{C}=\mathrm{O}) .{ }^{1} \mathrm{H}$ NMR $\left(400 \mathrm{MHz}, \mathrm{DMSO}-\mathrm{d}_{6}\right): \delta_{\mathrm{H}}$ $3.66\left(\mathrm{~s}, 3 \mathrm{H}, \mathrm{OCH}_{3}\right), 3.68\left(\mathrm{~s}, 3 \mathrm{H}, \mathrm{OCH}_{3}\right), 3.70(\mathrm{~d}, 1 \mathrm{H}, J=7.2 \mathrm{~Hz}$ pyrimidine-H), 3.73 (d, $1 \mathrm{H}, J=7.2 \mathrm{~Hz}$, pyrimidine-H), 7.15-7.20 (m, 2H, Ar-H), 7.39-7.48 (m, 4H, Ar-H and thiophene H-3,4,5), 9.90 (brs, 1H, NH), 10.25 (brs, 1H, NH). Anal. calcd. for $\mathrm{C}_{16} \mathrm{H}_{16} \mathrm{~N}_{2} \mathrm{O}_{3} \mathrm{~S}_{2}$ (348.44): C, 55.15; H, 4.63; N, 8.04. Found: C, $54.98 ; \mathrm{H}, 4.50 ; \mathrm{N}, 8.15$.

\section{4-(Thiophen-2-yl)-2-thioxo-6-(3,4,5- trimethoxyphenyl)tetrahydropyrimidin-5(6H)-one $(8 \mathrm{c})$}

Yield 63\%, m.p. $110-112{ }^{\circ} \mathrm{C}$, yellow needles. IR (KBr) $v$, $\mathrm{cm}^{-1}: 3318(\mathrm{NH}), 1716(\mathrm{C}=\mathrm{O}) .{ }^{1} \mathrm{H}$ NMR $\left(400 \mathrm{MHz}, \mathrm{DMSO}-\mathrm{d}_{6}\right): \delta_{\mathrm{H}}$ $3.64\left(\mathrm{~s}, 3 \mathrm{H}, \mathrm{OCH}_{3}\right), 3.66\left(\mathrm{~s}, 6 \mathrm{H}, 2 \mathrm{OCH}_{3}\right), 3.84(\mathrm{~d}, 1 \mathrm{H}, J=6.8 \mathrm{~Hz}$, pyrimidine- $\mathrm{H}), 3.87(\mathrm{~d}, 1 \mathrm{H}, J=6.8 \mathrm{~Hz}$, pyrimidine- $\mathrm{H}), 6.55$ (s, 2H, Ar-H), 7.01-7.06 (m, 2H, thiophene H-3,4), 7.49 (d, 1H, J = $7.5 \mathrm{~Hz}$, thiophene H-5), 9.85 (brs, $1 \mathrm{H}, \mathrm{NH}$ ), 10.24 (brs, $1 \mathrm{H} \mathrm{NH}$ ). ${ }^{13} \mathrm{C}$ NMR $\left(75 \mathrm{MHz}, \mathrm{DMSO}-\mathrm{d}_{6}\right): \delta_{\mathrm{c}} 54.3\left(2 \mathrm{CH}_{3} \mathrm{O}\right), 57.6\left(\mathrm{CH}_{3} \mathrm{O}\right)$, 60.5 (pyrimidine-C), 60.7 (pyrimidine-C), 107.1, 124.6, 125.3, $126.5,127.2,130.2,131.4,136.9,152.8$ (Ar-C and thiophene-C), 176.9, $191.2(\mathrm{C}=\mathrm{O}$ and $\mathrm{C}=\mathrm{S}) . \mathrm{MS}, \mathrm{m} / z(\%)\left(378,1.17 \%, \mathrm{M}^{+}\right)$. Anal. calcd. for $\mathrm{C}_{17} \mathrm{H}_{18} \mathrm{~N}_{2} \mathrm{O}_{4} \mathrm{~S}_{2}$ (378.46): C, 53.95; H, 4.79; N, 7.40. Found: C, 53.73; H, 4.61; N, 7.58.

\section{General method for preparation of compounds 9a-c}

To the thiopyrimidine derivatives $8 \mathbf{8 a - c}(10 \mathrm{mmole})$ was added chloroacetic acid (10 mmole) and the mixture was heated under reflux (TLC control; ethyl acetate/pet. ether: 1/3) till perfecting of reaction. The mixture was poured into ice-water, filtered, and then re-crystallization from acetic acid/water afforded the thiazolopyrimidines 9a-c, respectively.

\section{5-Phenyl-7-(thiophen-2-yl)-2H-thiazolo[3,2-a]pyrimidine- 3,6(5H,7H)-dione $(9 a)$}

Yield $85 \%$, m.p. $105-107{ }^{\circ} \mathrm{C}$, reaction time; 3 hours, yellow needles. IR (KBr) v, $\mathrm{cm}^{-1}$ : $1708(\mathrm{C}=\mathrm{O}), 1680(\mathrm{C}=\mathrm{O}) .{ }^{1} \mathrm{H}$ NMR (400 MHz, DMSO-d $\left.{ }_{6}\right): \delta_{\mathrm{H}} 3.64\left(\mathrm{~s}, 2 \mathrm{H}, \mathrm{CH}_{2}\right), 3.80(\mathrm{~s}, 1 \mathrm{H}$, 
pyrimidine-H), 3.85 (s, 1H, pyrimidine-H), 7.18 (d, 2H, $J=7.8$ $\mathrm{Hz}, \mathrm{Ar}-\mathrm{H}), 7.25(\mathrm{~m}, 2 \mathrm{H}, \mathrm{Ar}-\mathrm{H}), 7.56-7.65$ (m, 4H, Ar-H and thiohene $\mathrm{H}-3,4,5)$. MS, $m / z(\%)\left(327,2.2 \%, \mathrm{M}^{+}-1\right)$. Anal. calcd. for $\mathrm{C}_{16} \mathrm{H}_{12} \mathrm{~N}_{2} \mathrm{O}_{2} \mathrm{~S}_{2}$ (328.40): C, 58.52; H, 3.68; N, 8.53. Found: C, $58.69 ; \mathrm{H}, 3.77$; N, 8.39.

\section{5-(3,4-Dimethoxyphenyl)-7-(thiophen-2-yl)-2H-thiazolo[3,2- a]pyrimidine-3,6(5H,7H)-dione $(9 \mathrm{~b})$}

Yield 76\%, m.p. $110-112{ }^{\circ} \mathrm{C}$, reaction time; 4 hours, black powder. IR $(\mathrm{KBr}) v, \mathrm{~cm}^{-1}: 1705(\mathrm{C}=\mathrm{O}), 1680(\mathrm{C}=\mathrm{O}) .{ }^{1} \mathrm{H}$ NMR (400 MHz, DMSO-d $\left.)_{6}\right): \delta_{\mathrm{H}} 3.33,3.36\left(2 \mathrm{~s}, 6 \mathrm{H}, 2 \mathrm{OCH}_{3}\right), 3.72$ $\left(\mathrm{s}, 2 \mathrm{H}, \mathrm{CH}_{2}\right), 3.86(\mathrm{~s}, 1 \mathrm{H}$, pyrimidine- $\mathrm{H}), 3.90$ (s, $1 \mathrm{H}$, pyrimidineH), 6.91-6.97 (m, 2H, Ar-H), 7.06 (d, 1H, J= 7.8 Hz, Ar-H), 7.107.15 (m, 2H, thiophene H-3,4), $7.29(\mathrm{~d}, 1 \mathrm{H}, J=7.4 \mathrm{~Hz}$, thiophene $\mathrm{H}-5)$. MS, $m / z$, (\%) $\left(388,2.9 \%, \mathrm{M}^{+}\right)$. Anal. calcd. for $\mathrm{C}_{18} \mathrm{H}_{16} \mathrm{~N}_{2} \mathrm{O}_{4} \mathrm{~S}_{2}$ (388.46): C, 55.66; H, 4.15; N, 7.21. Found: C, 55.39; H, 4.05; N, 7.35 .

\section{7-(Thiophen-2-yl)-5-(3,4,5-trimethoxyphenyl)-2H-thiazolo[3,2- a]pyrimidine-3,6(5H,7H)-dione $(9 c)$}

Yield 79\%, m.p. $117-118{ }^{\circ} \mathrm{C}$, reaction time; 5 hours, brownish powder. IR (KBr) v, $\mathrm{cm}^{-1}: 1718(\mathrm{C}=\mathrm{O}), 1647(\mathrm{C}=\mathrm{O}) .{ }^{1} \mathrm{H}$ NMR (400 MHz, DMSO-d $\left.{ }_{6}\right): \delta_{\mathrm{H}} 3.58\left(\mathrm{~s}, 6 \mathrm{H}, 2 \mathrm{OCH}_{3}\right), 3.64$ (s, $\left.3 \mathrm{H}, \mathrm{OCH}_{3}\right), 3.78\left(\mathrm{~s}, 2 \mathrm{H}, \mathrm{CH}_{2}\right), 3.81(\mathrm{~s}, 1 \mathrm{H}$, pyrimidine- $\mathrm{H}), 3.85$ (s, 1H, pyrimidine-H), $6.86(\mathrm{~s}, 2 \mathrm{H}, \mathrm{Ar}-\mathrm{H}), 7.07-7.12(\mathrm{~m}, 2 \mathrm{H}$, thiophene $\mathrm{H}-3,4), 7.19$ (d, $1 \mathrm{H}, J=7.5 \mathrm{~Hz}$, thiophene H-5). MS, $m / z \quad(\%)\left(418,1.3 \%, \mathbf{M}^{+}\right)$. Anal. calcd. for $\mathrm{C}_{19} \mathrm{H}_{18} \mathrm{~N}_{2} \mathrm{O}_{5} \mathrm{~S}_{2}$ (418.48): C, 54.53; H, 4.34; N, 6.69. Found: C, 54.30; H, 4.25; N, 6.82 .

\section{General methods for preparation of compounds $10 \mathrm{~b}, \mathrm{c}$}

A mixture of the thiopyrimidine $8 \mathbf{b}, \mathbf{c}$ (10 mmole), 2chloroacetic acid (10 mmole) and acetic anhydride $(10 \mathrm{~mL})$ in acetic acid $(20 \mathrm{~mL})$ was stirred for 15 minutes, then the corresponding aromatic aldehyde $(10 \mathrm{mmole})$ and dry $\mathrm{CH}_{3} \mathrm{COONa}$ $(2 \mathrm{~g})$ were provided separately. The reaction components were refluxed for $4 \mathrm{~h}$ at which TLC showed completion of the reaction. The flask content was then added portion-wise to crushed ice and the precipitated solid was collected washed with water then recrystallized from acetic acid/water, to afford compound $\mathbf{1 0 b}, \mathbf{c}$, respectively.

\section{2-(3,4-Dimethoxybenzylidene)-5-(3,4-dimethoxyphenyl)-7- (thiophen-2-yl)-2H-thiazolo[3,2-a]pyrimidine-3,6(5H,7H)-dione (10b)}

Yield $83 \%$, m.p. $120-122{ }^{\circ} \mathrm{C}$, brownish powder. IR (KBr) v, $\mathrm{cm}^{-1}: 1712(\mathrm{C}=\mathrm{O}), 1674(\mathrm{C}=\mathrm{O}) .{ }^{1} \mathrm{H}$ NMR (400 MHz, DMSO$\left.\mathrm{d}_{6}\right): \delta_{\mathrm{H}} 3.69,3.71,3.74,3.77\left(4 \mathrm{~s}, 12 \mathrm{H}, 4 \mathrm{OCH}_{3}\right), 3.83(\mathrm{~s}, 1 \mathrm{H}$, pyrimidine-H), $3.86(\mathrm{~s}, 1 \mathrm{H}$, pyrimidine-H), 6.72-6.77 (m, 2H, Ar$\mathrm{H}), 6.79(\mathrm{~s}, 1 \mathrm{H}, \mathrm{CH}=\mathrm{C}), 6.83-7.12(\mathrm{~m}, 6 \mathrm{H}, \mathrm{Ar}-\mathrm{H}$ and thiophene $\mathrm{H}-$ 3,4), $7.85(\mathrm{~d}, 1 \mathrm{H}, J=7.5 \mathrm{~Hz}$, thiophene H-5). Anal. calcd. for
$\mathrm{C}_{27} \mathrm{H}_{24} \mathrm{~N}_{2} \mathrm{O}_{6} \mathrm{~S}_{2}$ (536.62): C, 60.43; H, 4.51; N, 5.22. Found: $\mathrm{C}$, $60.29 ; \mathrm{H}, 4.35 ; \mathrm{N}, 5.32$.

7-(Thiophen-2-yl)-2-(3,4,5-trimethoxybenzylidene)-5-(3,4,5trimethoxyphenyl)-2H-thiazolo[3,2-a]pyrimidine-3,6(5H,7H)dione $(10 c)$

Yield $83 \%$, m.p. $115-117^{\circ} \mathrm{C}$, yellow needles. IR (KBr) v, $\mathrm{cm}^{-1}: 1718(\mathrm{C}=\mathrm{O}), 1647(\mathrm{C}=\mathrm{O}) .{ }^{1} \mathrm{H}$ NMR (400 MHz, DMSO-d 6 ): $\delta_{\mathrm{H}} 3.53\left(\mathrm{~s}, 6 \mathrm{H}, 2 \mathrm{OCH}_{3}\right), 3.57\left(\mathrm{~s}, 6 \mathrm{H}, 2 \mathrm{OCH}_{3}\right), 3.60(\mathrm{~s}, 6 \mathrm{H}, 2 \mathrm{O}$ $\left.\mathrm{CH}_{3}\right), 3.76(\mathrm{~s}, 1 \mathrm{H}$, pyrimidine- $\mathrm{H}), 3.80(\mathrm{~s}, 1 \mathrm{H}$, pyrimidine- $\mathrm{H}), 6.39$ (s, 2H, Ar-H), 7.32-7.38 (m, 4H, Ar-H, CH=C and thiophene-H), $7.51(\mathrm{~d}, J=7.4 \mathrm{~Hz}, 1 \mathrm{H}$, thiophene-H), $7.72(\mathrm{~d}, 1 \mathrm{H}, J=7.6 \mathrm{~Hz}$, thiophene-H). Anal. calcd. for $\mathrm{C}_{29} \mathrm{H}_{28} \mathrm{~N}_{2} \mathrm{O}_{8} \mathrm{~S}_{2}$ (596.67): C, 58.38; H, 4.73; N, 4.70. Found: C, 58.61; H, 4.81; N, 4.48.

\section{General procedure for the preparation of compounds 11a-c}

To a suspension of compounds $2 \mathbf{a}-\mathbf{c}$ (10 mmole) and thiosemicarbazide $(10 \mathrm{mmole})$ in ethanol $(20 \mathrm{~mL})$ was added portion wise potassium hydroxide $(1.5 \mathrm{~g})$ then the mixture was heated under efflux till completion of the reaction (TLC) (ethyl acetate/pet. ether: 1/3). Cold diluted $\mathrm{HCl}(15 \mathrm{~mL})$ was then added drop wise in an ice bath. The appeared precipitated solid $\mathrm{m}$ was filtered and re-crystallized from proper solvent.

\section{1-Amino-6-phenyl-4-(thiophen-2-yl)-2- thioxotetrahydropyrimidin-5(6H)-one (11a)}

Yield 73\%, m.p. $140-142{ }^{\circ} \mathrm{C}$, reaction time; 1 hour, solvent of crystallization: ethanol/water (2:1), yellow powder. IR (KBr) v, $\mathrm{cm}^{-1}:$ 3420-3363 (NH and $\left.\mathrm{NH}_{2}\right), 1727(\mathrm{C}=\mathrm{O}) .{ }^{1} \mathrm{H}$ NMR $\left(400 \mathrm{MHz}, \mathrm{DMSO}-\mathrm{d}_{6}\right): \delta_{\mathrm{H}} 3.62(\mathrm{~s}, 1 \mathrm{H}$, pyrimidine-H), $3.67(\mathrm{~s}, 1 \mathrm{H}$, pyrimidine-H), 5.89 (brs, $2 \mathrm{H}, \mathrm{NH}_{2}$ ), 6.62-6.68 (m, 2H, Ar-H), 7.26 $(\mathrm{d}, 1 \mathrm{H}, J=7.5 \mathrm{~Hz}, \mathrm{Ar}-\mathrm{H}), 7.71-7.79(\mathrm{~m}, 5 \mathrm{H}, \mathrm{Ar}-\mathrm{H}$ and thiohene H3,4,5), 9.80 (brs, $1 \mathrm{H}, \mathrm{NH}$ ). Anal. calcd. for $\mathrm{C}_{14} \mathrm{H}_{13} \mathrm{~N}_{3} \mathrm{OS}_{2}$ (303.40): C, 55.42; H, 4.32; N, 13.85. Found: 55.58; H, 4.47; N, 13.66.

\section{1-Amino-6-(3,4-dimethoxyphenyl)-4-(thiophen-2-yl)-2- thioxotetrahydropyrimidin-5(6H)-one (11b)}

Yield $85 \%$, m.p. $118-120{ }^{\circ} \mathrm{C}$, reaction time; 30 minutes, solvent of crystallization: ethanol/water (1:1), yellow powder. IR (KBr) v, cm ${ }^{-1}$ : 3430-3325 ( $\mathrm{NH}$ and $\left.\mathrm{NH}_{2}\right), 1727(\mathrm{C}=\mathrm{O}) .{ }^{1} \mathrm{H}$ NMR $\left(400 \mathrm{MHz}, \mathrm{DMSO}-\mathrm{d}_{6}\right): \delta_{\mathrm{H}} 3.33,3.36\left(2 \mathrm{~s}, 6 \mathrm{H}, 2 \mathrm{OCH}_{3}\right), 3.60$ (s, $1 \mathrm{H}$, pyrimidine- $\mathrm{H}), 3.64$ (s, $1 \mathrm{H}$, pyrimidine- $\mathrm{H}), 5.90$ (brs, $2 \mathrm{H}$, $\mathrm{NH}_{2}$ ), 6.68 (s, 1H, Ar-H), 6.74-6.79 (m, 2H, Ar-H), 6.99-7.08 (m, $1 \mathrm{H}$, thiohene-H), 7.35 (d, $1 \mathrm{H}, J=7.2 \mathrm{~Hz}$, thiohene-H), 7.39 (d, $1 \mathrm{H}, J=7.5 \mathrm{~Hz}$, thiohene-H), 10.02 (brs, $1 \mathrm{H}, \mathrm{NH}) . \mathrm{MS}, \mathrm{m} / z(\%)$ (363.1, 4.5\%, $\mathrm{M}^{+}$). Anal. calcd. for $\mathrm{C}_{16} \mathrm{H}_{17} \mathrm{~N}_{3} \mathrm{O}_{3} \mathrm{~S}_{2}$ (363.45): C, 52.88; H, 4.71; N, 11.56. Found: C, 52.69; H, 4.55; N, 11.71.

\section{1-Amino-4-(thiophen-2-yl)-2-thioxo-6-(3,4,5- trimethoxyphenyl)tetrahydro-pyrimidin-5(6H)-one (11c)}

Yield $72 \%$, m.p. $130-132{ }^{\circ} \mathrm{C}$, reaction time; 25 minutes, solvent of crystallization: ethanol/water $(1: 1)$, yellow powder. IR $(\mathrm{KBr}) v, \mathrm{~cm}^{-1}:$ 3420-3310 (NH and $\left.\mathrm{NH}_{2}\right), 1704(\mathrm{C}=\mathrm{O}) .{ }^{1} \mathrm{H}$ NMR $\left(400 \mathrm{MHz}, \mathrm{DMSO}-\mathrm{d}_{6}\right): \delta_{\mathrm{H}} 3.77\left(\mathrm{~s}, 6 \mathrm{H}, 2 \mathrm{OCH}_{3}\right), 3.81(\mathrm{~s}, 3 \mathrm{H}$ 
$\left.\mathrm{OCH}_{3}\right), 4.50(\mathrm{~s}, 1 \mathrm{H}$, pyrimidine- $\mathrm{H}), 4.54$ (s, 1H, pyrimidine- $\left.\mathrm{H}\right)$, 5.88 (brs, 2H, $\left.\mathrm{NH}_{2}\right), 6.60(\mathrm{~s}, 2 \mathrm{H}, \mathrm{Ar}-\mathrm{H}), 6.95-7.01(\mathrm{~m}, 2 \mathrm{H}$, thiohene $\mathrm{H}-3,4), 7.39$ (d, $1 \mathrm{H}, J=7.8 \mathrm{~Hz}$, thiohene $\mathrm{H}-5), 10.05$ (brs, $1 \mathrm{H}, \mathrm{NH})$. MS, $m / z(\%)\left(393,1.27 \%, \mathrm{M}^{+}\right)$. Anal. calcd. for $\mathrm{C}_{17} \mathrm{H}_{19} \mathrm{~N}_{3} \mathrm{O}_{4} \mathrm{~S}_{2}$ (393.48): C, 51.89; H, 4.87; N, 10.68. Found: C, $51.70 ; \mathrm{H}, 4.92 ; \mathrm{N}, 10.79$.

\section{General procedure for the preparation of compounds 12a,c}

A mixture of the epoxide derivatives $\mathbf{2 a , c}$ (10 mmole), urea $(10 \mathrm{mmole})$ and anhydrous $\mathrm{KOH}$ pellets $(0.5 \mathrm{~g})$ in absolute ethyl alcohol $(10 \mathrm{~mL})$ was heated at reflux temperature for 45 minutes. The reaction mixture poured on cold hydrochloric acid (15 mL), filtered, dried and crystallized from ethanol/water (1:1).

\section{4-Phenyl-6-(thiophen-2-yl)tetrahydropyrimidine-2,5-dione (12a)}

Yield $78 \%$, m.p. $150-152{ }^{\circ} \mathrm{C}$, yellow needles. IR (KBr) v, $\mathrm{cm}^{-1}$ : $3320(\mathrm{NH}), 1700(\mathrm{C}=\mathrm{O}), 1658(\mathrm{C}=\mathrm{O}) .{ }^{1} \mathrm{H}$ NMR $(400 \mathrm{MHz}$, DMSO-d $\left.{ }_{6}\right): \delta_{\mathrm{H}} 3.85(\mathrm{~d}, 1 \mathrm{H}, J=6.8 \mathrm{~Hz}$, pyrimidine-H), $3.89(\mathrm{~d}, 1 \mathrm{H}$, $J=6.8 \mathrm{~Hz}$, pyrimidine-H), 7.22-7.28 (m, 4H, Ar-H), 7.79-7.90 (m, 4H, Ar-H and thiophene H-3,4,5), 9.50 (brs, 1H, NH), 10.12 (brs, $1 \mathrm{H}, \mathrm{NH}) .{ }^{13} \mathrm{C}$ NMR $\left(75 \mathrm{MHz}, \mathrm{DMSO}-\mathrm{d}_{6}\right): \delta_{\mathrm{c}} 70.5$ (pyrimidine C), 71.9 (pyrimidine C), 112.1, 127.5, 128.2, 132.1, 135.8, 137.9, 140.5, 152.4 (Ar-C and thiphene-C), 161.1, 179.9 (2C=O). Anal. calcd. for $\mathrm{C}_{14} \mathrm{H}_{12} \mathrm{~N}_{2} \mathrm{O}_{2} \mathrm{~S}$ (272.32): C, 61.75; H, 4.44; N, 10.29. Found: C, 61.94; H, 4.59; N, 10.18 .

\section{4-(Thiophen-2-yl)-6-(3,4,5-trimethoxyphenyl) tetrahydropyrimidine 0 2,5-dione (12c)}

Yield 78\%, m.p. 95-97 ${ }^{\circ} \mathrm{C}$, yellow needles. IR (KBr) v, $\mathrm{cm}^{-1}$ : $3267(\mathrm{NH}), 1708(\mathrm{C}=\mathrm{O}), 1653(\mathrm{C}=\mathrm{O}) .{ }^{1} \mathrm{H}$ NMR $(400 \mathrm{MHz}$, DMSO-d $\left.{ }_{6}\right): \delta_{\mathrm{H}} 3.31\left(\mathrm{~s}, 6 \mathrm{H}, 2 \mathrm{OCH}_{3}\right), 3.35\left(\mathrm{~s}, 3 \mathrm{H}, \mathrm{OCH}_{3}\right), 3.91(\mathrm{~d}$, $1 \mathrm{H}, J=6.8 \mathrm{~Hz}$, pyrimidine-H), $3.94(\mathrm{~d}, 1 \mathrm{H}, J=6.8 \mathrm{~Hz}$, pyrimidine-H), $7.22(\mathrm{~s}, 2 \mathrm{H}, \mathrm{Ar}-\mathrm{H}), 7.50-7.55(\mathrm{~m}, 2 \mathrm{H}$, thiohene $\mathrm{H}$ 3,4), 7.78 (d, 1H, $J=7.2 \mathrm{~Hz}$, thiohene $\mathrm{H}-5$ ), 8.72 (brs, 2H, 2NH). MS, $m / z(\%)\left(362.2,3.10 \%, \mathrm{M}^{+}\right)$. Anal. calcd. for $\mathrm{C}_{17} \mathrm{H}_{18} \mathrm{~N}_{2} \mathrm{O}_{5} \mathrm{~S}$ (362.40): C, 56.34; H, 5.01; N, 7.73. Found: C, 56.19; H, 5.16; N, 7.88 .

\section{2-Imino-4-phenyl-6-(thiophen-2-yl)-tetrahydropyrimidin- 5(2H)one (13a)}

A mixture of compound 2a (10 mmole), guanidine hydrochloride (10 mmole), potassium hydroxide (1.0 g) in ethanol $(25 \mathrm{~m})$ was heated at reflux temperature for 3 hours. The solvent was evaporated till dryness and then washed with cold methanol. Yield 50\%, m.p. $270-272{ }^{\circ} \mathrm{C}$, white powder. IR $(\mathrm{KBr}) v, \mathrm{~cm}^{-1}$ : $3379(\mathrm{NH}), 1712(\mathrm{C}=\mathrm{O}) .{ }^{1} \mathrm{H}$ NMR $\left(400 \mathrm{MHz}, \mathrm{DMSO}_{-}\right): \delta_{\mathrm{H}} 3.70$ (s, $1 \mathrm{H}$, pyrimidine- $\mathrm{H}), 3.73(\mathrm{~s}, 1 \mathrm{H}$, pyrimidine- $\mathrm{H}), 7.22(\mathrm{~d}, 2 \mathrm{H}, J=$ 7.5 Hz, Ar-H), 7.26-7.31 (m, 2H, Ar-H), 7.57-7.63 (m, 4H, Ar-H and thiophene $\mathrm{H}-3,4,5$ ), 9.72-9.76 (brs, $2 \mathrm{H}, 2 \mathrm{NH}$ ), 10.84 (brs, $1 \mathrm{H}$, $\mathrm{NH}$ ). Anal. calcd. for $\mathrm{C}_{14} \mathrm{H}_{13} \mathrm{~N}_{3} \mathrm{OS}$ (271.34): C, 61.97; H, 4.83; N, 15.49. Found: C, 62.12; H, 4.91; N, 15.29.
General method for preparation of compounds $14 \mathrm{~b}, \mathrm{c}$

A mixture of compound $\mathbf{2 b , c}$ (10 mmole), carbon disulfide (10 mmole), potassium hydroxide $(1 \mathrm{~g})$ in ethanol (20 $\mathrm{mL}$ ) was refluxed for 3 hours. The mixture was put onto crushed ice-water, filtered and crystallized.

(4-(3,4-Dimethoxyphenyl)-2-thioxo-1,3-oxathiolan-5yl)(thiophen-2-yl)methanone (14b)

Yield $78 \%$, m.p. $180-182{ }^{\circ} \mathrm{C}$, solvent of crystallization: ethanol/water $(1: 1)$, white powder. IR $(\mathrm{KBr}) \mathrm{v}, \mathrm{cm}^{-1}: 1702(\mathrm{C}=\mathrm{O})$. ${ }^{1} \mathrm{H}$ NMR (400 MHz, DMSO-d 6 ): $\delta_{\mathrm{H}} 3.89,3.92\left(2 \mathrm{~s}, 6 \mathrm{H}, 2 \mathrm{O} \mathrm{CH}_{3}\right)$, $4.21(\mathrm{~d}, 1 \mathrm{H}, J=6.8 \mathrm{~Hz}$, oxathiolan-H), $4.57(\mathrm{~d}, 1 \mathrm{H}, J=6.8 \mathrm{~Hz}$, oxathiolan-H), 7.19 (d, $1 \mathrm{H}, J=8.2 \mathrm{~Hz}, \mathrm{Ar}-\mathrm{H}), 7.27-7.32(\mathrm{~m}, 2 \mathrm{H}$, Ar-H), 7.66-7.71 (m, 2H, thiophene H-3,4), 7.77 (d, 1H, $J=7.4$ $\mathrm{Hz}$, thiophene H-5). Anal. calcd. for $\mathrm{C}_{16} \mathrm{H}_{14} \mathrm{O}_{4} \mathrm{~S}_{3}$ (366.46): C, 52.44; H, 3.85. Found: C, 52.58; H, 3.70.

\section{Thiophen-2-yl(2-thioxo-4-(3,4,5-trimethoxyphenyl)-1,3- oxathiolan-5-yl)methanone (14c)}

Yield $82 \%$, m.p. $160-162{ }^{\circ} \mathrm{C}$, solvent of crystallization: ethanol, yellow crystals. IR (KBr) v, $\mathrm{cm}^{-1}: 1710(\mathrm{NH}), 1713$ $(\mathrm{C}=\mathrm{O}) .{ }^{1} \mathrm{H}$ NMR $\left(400 \mathrm{MHz}, \mathrm{DMSO}-\mathrm{d}_{6}\right): \delta_{\mathrm{H}} 3.88\left(\mathrm{~s}, 6 \mathrm{H}, 2 \mathrm{OCH}_{3}\right)$, $3.90\left(\mathrm{~s}, 3 \mathrm{H}, \mathrm{OCH}_{3}\right), 4.34(\mathrm{~d}, 1 \mathrm{H}, J=6.6 \mathrm{~Hz}$, oxathiolan-H), 4.85 (d, $1 \mathrm{H}, J=6.6 \mathrm{~Hz}$, oxathiolan-H), 7.19 (s, 2H, Ar-H), 7.26-7.31 (m, 2H, thiophene $\mathrm{H}-3,4), 7.77(\mathrm{~d}, 1 \mathrm{H}, J=6.8 \mathrm{~Hz}$, thiophene $\mathrm{H}-5)$. ${ }^{13} \mathrm{C}$ NMR (75 MHz, DMSO-d 6$): \delta_{\mathrm{c}} 56.1\left(2 \mathrm{OCH}_{3}\right), 59.2\left(\mathrm{OCH}_{3}\right)$, 60.9 (oxathiolan-C), 105.7 (oxathiolan-C), 120.9, 123.4, 125.3, 128.2, 131.7, 133.8, 140.5, 145.5 (Ar-C and thiophene-C), 173.5 $(\mathrm{C}=\mathrm{O}), 181.9(\mathrm{C}=\mathrm{S})$. MS, $\mathrm{m} / z(\%)\left(396.50,1.10 \%, \mathrm{M}^{+}\right)$. Anal. calcd. for $\mathrm{C}_{17} \mathrm{H}_{16} \mathrm{O}_{5} \mathrm{~S}_{3}$ (396.49): C, 51.50; H, 4.07. Found: C, 51.38; $\mathrm{H}, 3.95$.

\section{General method for preparation of compounds 15a,b}

A solution of the acetylated bromoglucose or acetylated bromoxylose $(5 \mathrm{mmole})$ in acetone $(15 \mathrm{~mL})$ was inserted progressively to a stirred mixture of compound $\mathbf{8 a}(5 \mathrm{mmole})$ and $\mathrm{KOH}$ ( $5 \mathrm{mmole})$ in water $(2 \mathrm{~mL})$. Stirring was continued at r.t. for a time at which reaction was judged complete by TLC (chloroform/methanol; 99.7/0.3 v/v). Evaporation of the solvent resulted in a residue that was washed with water $(10 \mathrm{~mL})$. The organic layer was extracted with chloroform, dried and evaporated under reduced pressure. Petroleum ether (b.p. $\left.40-60{ }^{\circ} \mathrm{C}\right)(45 \mathrm{~mL})$ was then provided to the remnant with stirring. The resulted solid was filtered and re-crystallized.

\section{4-Phenyl-6-(thiophen-2-yl)-2-(2,3,4-tri-O-acetyl-D- glucopyranosylthio)tetrahydro-pyrimidin-5(6H)-one (15a)}

Yield 83\%, m.p.142-144 ${ }^{\circ} \mathrm{C}$, reaction time; 3 hours, solvent of crystallization: ethanol/water (3:1), pale yellow crystals. IR (KBr) v, $\mathrm{cm}^{-1}: 3420(\mathrm{NH}), 1703(\mathrm{C}=\mathrm{O}), 1742(\mathrm{C}=\mathrm{O}) .{ }^{1} \mathrm{H}$ NMR $\left(400 \mathrm{MHz}, \mathrm{CDCl}_{3}\right): \delta_{\mathrm{H}} 1.92,1.96,1.98,2.01\left(4 \mathrm{~s}, 12 \mathrm{H}, 4 \mathrm{CH}_{3} \mathrm{CO}\right)$, $3.62(\mathrm{~m}, 1 \mathrm{H}, \mathrm{H}-5), 3.78$ (s, 1H, pyrimidine-H), $3.88(\mathrm{dd}, 1 \mathrm{H}, J=$ $3.6 \mathrm{~Hz}, J=11.2 \mathrm{~Hz}, \mathrm{H}-6$ '), 4.20 (dd, $1 \mathrm{H}, J=3.2, J=11.2 \mathrm{~Hz}, \mathrm{H}-$ 6"), 4.38 (s, 1H, pyrimidine-H), 4.57 (t, 1H, J=9.4 Hz, H-4'), 4.95 
(t, 1H, $\left.J=9.8 \mathrm{~Hz}, \mathrm{H}-2^{\prime}\right), 5.23\left(\mathrm{~d}, 1 \mathrm{H}, J=9.8 \mathrm{~Hz}, \mathrm{H}-1^{\prime}\right), 5.39$ (t, $\left.1 \mathrm{H}, J=9.4 \mathrm{~Hz}, \mathrm{H}-3^{\prime}\right), 6.76-6.84(\mathrm{~m}, 4 \mathrm{H}, \mathrm{Ar}-\mathrm{H}), 7.12-7.25$ (m, 4H, Ar-H and thiophene $\mathrm{H}-3,4,5), 7.93$ (brs, $1 \mathrm{H}, \mathrm{NH}) .{ }^{13} \mathrm{C} \mathrm{NMR}(75$ $\left.\mathrm{MHz}, \mathrm{CDCl}_{3}\right): \delta_{\mathrm{c}} 19.8,20.1,20.4\left(4 \mathrm{CH}_{3} \mathrm{CO}\right), 61.2(\mathrm{C}-6$ '), $64.1(\mathrm{C}-$ 4'), 65.2 (pyrimidine-C), 66.3 (pyrimidine-C), 68.2 (C-3'), 71.1 (C2'), 71.8 (C-5'), 89.6 (C-1'), 110.5, 119.2, 120.6, 122.4, 125.8, 129.3, 130.3, 134.9, 155.4 (Ar-C, thiophene-C and pyrimidine $\mathrm{C}$ ), 170.1, 170.8, 173.1, $179.5(5 \mathrm{C}=\mathrm{O})$. Anal. calcd. for $\mathrm{C}_{28} \mathrm{H}_{30} \mathrm{~N}_{2} \mathrm{O}_{10} \mathrm{~S}_{2}$ (618.67): C, 54.36; H, 4.89; N, 4.53. Found: C, 54.20; H, 4.72; N, 4.69 .

4-Phenyl-6-(thiophen-2-yl)-2-(2,3,4-tri-O-acetyl-Dxylopyranosylthio)tetrahydro-pyrimidin-5(6H)-one (15b)

Yield $78 \%$, m.p. $149-151{ }^{\circ} \mathrm{C}$, reaction time; 4 hours, solvent of crystallization: ethanol/water (3:1), pale yellow powder. IR (KBr) v, $\mathrm{cm}^{-1}: 3367(\mathrm{NH}), 1718(\mathrm{C}=\mathrm{O}), 1750(\mathrm{C}=\mathrm{O}) .{ }^{1} \mathrm{H}$ NMR $\left(400 \mathrm{MHz}, \mathrm{CDCl}_{3}\right): \delta_{\mathrm{H}} 1.92,1.96,2.01\left(3 \mathrm{~s}, 9 \mathrm{H}, 3 \mathrm{CH}_{3} \mathrm{CO}\right), 3.82(\mathrm{~s}$, $1 \mathrm{H}$, pyrimidine-H), 3.86 (dd, $\left.1 \mathrm{H}, J=2.8 \mathrm{~Hz}, J=11.0 \mathrm{~Hz}, \mathrm{H}-5{ }^{\prime}\right)$, 4.16-4.18 (m, 1H, H-5"), 4.37 (s, 1H, pyrimidine-H), 4.59 (t, 1H, J $\left.=9.2 \mathrm{~Hz}, \mathrm{H}-4^{\prime}\right), 4.94$ (t, 1H, $\left.J=9.8 \mathrm{~Hz}, \mathrm{H}-2^{\prime}\right), 5.24$ (d, 1H, $J=9.8$ Hz, H-1'), 5.38 (t, 1H, J=9.2 Hz, H-3'), 6.89-7.02 (m, 4H, Ar-H), 7.14-7.27 (m, 4H, Ar-H and thiophene H-3,4,5), 7.98 (brs, 1H, $\mathrm{NH}$ ). Anal. calcd. for $\mathrm{C}_{25} \mathrm{H}_{26} \mathrm{~N}_{2} \mathrm{O}_{8} \mathrm{~S}_{2}$ (546.61): C, 54.93; H, 4.79; N, 5.13. Found: C, 54.71; H, 4.86; N, 4.98.

\section{General method for preparation of compounds 16a,b}

The acetylated thioglycosides 15a,b $(5 \mathrm{mmol})$ was dissolved in dry saturated methanolic ammonia solution $(20 \mathrm{~mL})$ and stirred at $0{ }^{\circ} \mathrm{C}$ for $1 \mathrm{~h}$, then stirring was persisted at r.t. for $5 \mathrm{~h}$. Removal of the solvent under vacuum at $40{ }^{\circ} \mathrm{C}$ gave a solid residue, which was recrystallized from ethanol to give the corresponding free glycoside $\mathbf{1 6 a , b}$.

\section{4-Phenyl-6-(thiophen-2-yl)-2-(D- glucopyranosylthio)tetrahydropyrimidin-5(6H)-one (16a)}

Yield $81 \%$, m.p. $188-190{ }^{\circ} \mathrm{C}$, brown powder. IR (KBr) v, $\mathrm{cm}^{-1}$ : 3520-3475 (OH), $3420(\mathrm{NH}), 1725(\mathrm{C}=\mathrm{O}) .{ }^{1} \mathrm{H}$ NMR (400 MHz, DMSO-d $\left.\mathrm{d}_{6}\right): \delta_{\mathrm{H}} 3.39$ (m, 2H, H-6',6"), 3.77-3.82 (m, 1H, H5'), 3.98-4.11 (m, 2H, H-4',3'), 4.20 (s, 1H, pyrimidine-H), 4.35 (s, $1 \mathrm{H}$, pyrimidine- $\mathrm{H}), 4.72-4.75\left(\mathrm{~m}, 2 \mathrm{H}, \mathrm{H}-2{ }^{\prime}\right.$ and $\left.\mathrm{OH}\right), 5.22-5.28(\mathrm{~m}$, $2 \mathrm{H}, 2 \mathrm{OH}), 5.40-5.43(\mathrm{~m}, 1 \mathrm{H}, \mathrm{OH}), 5.72$ (d, 1H, $J=9.6 \mathrm{~Hz}, \mathrm{H}-1$ '), 7.39-7.52 (m, 4H, Ar-H), 7.92-8.02 (m, 4H, Ar-H and thiophene $\mathrm{H}-3,4,5$ ), 9.02 (brs, $1 \mathrm{H}, \mathrm{NH}$ ). Anal. calcd. for $\mathrm{C}_{20} \mathrm{H}_{22} \mathrm{~N}_{2} \mathrm{O}_{6} \mathrm{~S}_{2}$ (450.52): C, 53.32; H, 4.92; N, 6.22. Found: C, 53.49; H, 5.01; N, 6.05 .

\section{4-Phenyl-6-(thiophen-2-yl)-2-(D- \\ xylopyranosylthio)tetrahydropyrimidin-5(6H)-one (16b)}

Yield $72 \%$, m.p. $193-195{ }^{\circ} \mathrm{C}$, brown powder. IR (KBr) v, $\mathrm{cm}^{-1}$ : 3510-3488 (OH), $3363(\mathrm{NH}), 1722(\mathrm{C}=\mathrm{O}) .{ }^{1} \mathrm{H}$ NMR (400 MHz, DMSO-d $\left.\mathrm{d}_{6}\right): \delta_{\mathrm{H}} 3.40-3.45(\mathrm{~m}, 2 \mathrm{H}, \mathrm{H}-5,5 "), 3.89-3.92(\mathrm{~m}, 1 \mathrm{H}$, H-4'), 4.12-4.15 (m, 1H, H-3'), 4.22 (s, 1H, pyrimidine-H), 4.38 (s, $1 \mathrm{H}$, pyrimidine- $\mathrm{H}), 4.75-4.51\left(\mathrm{~m}, 2 \mathrm{H}, \mathrm{H}-2^{\prime}\right.$ and $\left.\mathrm{OH}\right), 5.24-5.27(\mathrm{~m}$, $1 \mathrm{H}, \mathrm{OH}), 5.40-5.44(\mathrm{~m}, 1 \mathrm{H}, \mathrm{OH}), 5.74\left(\mathrm{~d}, 1 \mathrm{H}, J=9.8 \mathrm{~Hz}, \mathrm{H}-1^{\prime}\right)$,
7.47-7.58 (m, 4H, Ar-H), 7.75-7.86 (m, 4H, Ar-H and thiophene $\mathrm{H}-3,4,5$ ), 9.15 (brs, $1 \mathrm{H}, \mathrm{NH}$ ). Anal. calcd. for $\mathrm{C}_{19} \mathrm{H}_{20} \mathrm{~N}_{2} \mathrm{O}_{5} \mathrm{~S}_{2}$ (420.50): C, 54.27; H, 4.79; N, 6.66. Found: C, 54.03; H, 4.87; N, 6.49 .

\section{CYTOTOXIC ACTIVITY}

\section{Material}

The cell lines namely; Human liver carcinoma (HepG-2), PC-3 (human prostate adenocarcinoma) and HCT116 (human colorectal carcinoma) cell culture were brought from the American Type Culture Collection (Rockville, MD, USA).

\section{Cell culture}

The cells were preserved in the medium of Roswell Park Memorial Institute (RPMI-1640) that was replenished with $10 \%$ heat-inactivated FBS (fetal bovine serum), $100 \mathrm{U} / \mathrm{mL}$ penicillin and $100 \mathrm{U} / \mathrm{mL}$ streptomycin. The cells were grown at $37{ }^{\circ} \mathrm{C}$ in $5 \%$ $\mathrm{CO}_{2}$ and $95 \%$ moisture.

\section{MTT Cytotoxicity Assay}

The cytotoxic activity against the three cell lines was investigated by means of 3-[4,5-dimethyl-2-thiazolyl)-2,5diphenyl-2H-tetrazolium bromide (MTT) assay, which is founded on the incision of the tetrazolium salt by mitochondrial dehydrogenases in viable cells (Awad et al., 2014; Soliman et al., 2014).

The cells were distributed in a 96-well sterile microplate $\left(5 \times 10^{4}\right.$ cells/well), and incubated with each of synthesized compound or Doxorubicin ${ }^{\circledR}$ (positive control), prepared in a set of various concentrations in diemthyl sulfoxide, at $37{ }^{\circ} \mathrm{C}$ for 48 hours in a serum-free medium prior to the assay. The media were cautiously isolated after incubation, and then MTT $(2.5 \mathrm{mg} / \mathrm{mL}, 40$ $\mu \mathrm{L}$ ) was provided to each well and then incubated for further four hours. The crystals of purple formazan dye were solubilized by providing dimethyl sulfoxide $(200 \mu \mathrm{L})$. At $590 \mathrm{~nm}$ (SpectraMax ${ }^{\circledR}$ Paradigm ${ }^{\circledR}$ Multi-Mode microplate reader), the absorbance was determined. The average percentage of viable cells with respect to to the untreated control cells expresses the relative cell viability.

\section{Statistical Analysis}

All experimental investigations were performed in triplicate with repeating at three different days. All the OBTAINED values were expressed as mean $\pm \mathrm{SD} . \mathrm{IC}_{50} \mathrm{~s}$ have been determined by PROBIT analysis by means of SPSS software (version 20, SPSS Inc., Chicago, IL, USA).

\section{RESULTS AND DISCUSSION}

Chemistry

$\alpha, \beta$-Unsaturated ketones 1a-c were prepared by reaction of aromatic aldehyde (namely benzaldehyde, 3,4dimethoxybenzaldehyde, and 3,4,5-trimethoxybenzaldehyde) with 2-acetylthiophene. The corresponding epoxide derivatives 2a-c were prepared by reaction of $\alpha, \beta$-unsaturated ketones 1a-c with 
hydrogen peroxide in basic medium. Compounds 1a-c and 2a-c were used as starting key compounds for the preparation of a variety of substituted pyrimidine derivatives. Reaction of compounds 1a-c with thiourea in sodium hydroxide afforded the corresponding thiopyrimidine derivatives 3a-c. The signals assigned to the aryl, pyrimidine ring and $\mathrm{NH}$ protons in their ${ }^{1} \mathrm{H}$ NMR spectra confirmed the assigned structure. Thiopyrimidine derivatives $\mathbf{3 b}, \mathbf{c}$ were allowed to react with chloroacetic acid and resulted in the formation of thiazolo[3,2-a]pyrimidine derivatives $\mathbf{5 b}, \mathbf{c}$. The products revealed absorption band for carbonyl group in the infra-red spectrum and the corresponding ${ }^{1} \mathrm{H}$ NMR data revealed singlet peaks for $-\mathrm{SCH}_{2}$. Compounds $\mathbf{5 b}$,c were reacted with 3,4-dimethoxybenzaldehyde and 3,4,5trimethoxybenzaldehyde $(\mathbf{6 a}, \mathbf{b})$ and afforded the thiazolo[3,2a]pyrimidin-3(2H)-one $\mathbf{7 b}$, and $\mathbf{7 c}$, respectively. The ${ }^{1} \mathrm{H}$ NMR spectrum of compounds $\mathbf{7 b , c}$ revealed disappearance of singlet peak of the $-\mathrm{SCH}_{2}$ and other assignments greed with their structures. Reaction of compounds 1a-c with guanidine hydrochloride in potassium hydroxide afforded the dihydropyrimidin-2(1H)-imine derivative $4 \mathbf{c}$ (scheme 1 ). The resulting iminopyrimidine derivative $\mathbf{4 c}$ showed the assigned absorption bands for $\mathrm{NH}$ functions in the IR spectrum in addition to the doublet signals attributed to the hydrogens $(\mathrm{H}-4$ and $\mathrm{H}-5)$ in its ${ }^{1} \mathrm{H}$ NMR spectrum.

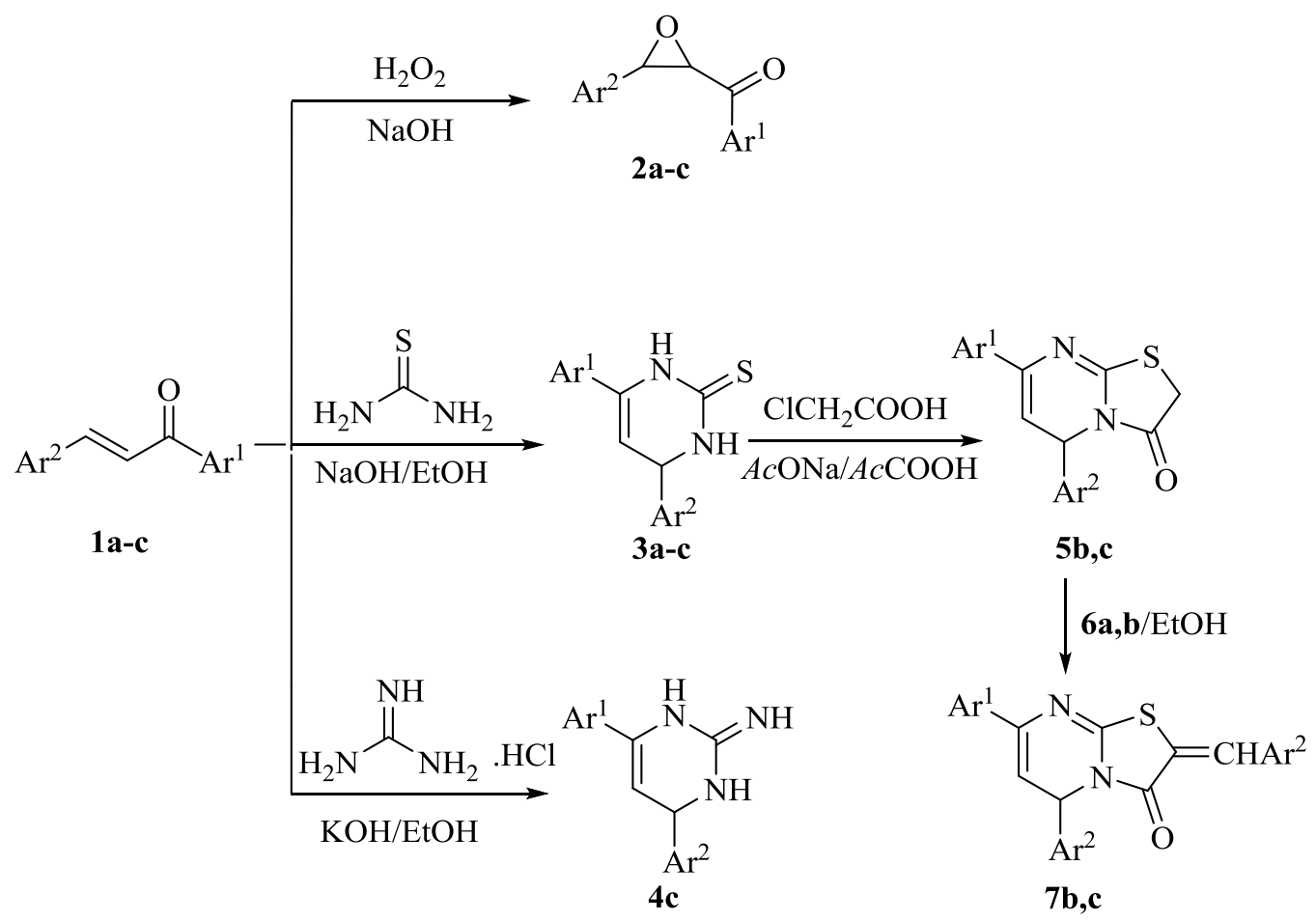

a; $\mathrm{Ar}^{1}=$ thiophen-2-yl, $\mathrm{Ar}^{2}=-\mathrm{C}_{6} \mathrm{H}_{5}$

b; $\mathrm{Ar}^{1}=$ thiophen-2-yl, $\mathrm{Ar}^{2}=3,4-\left(\mathrm{OCH}_{3}\right)_{2} \mathrm{C}_{6} \mathrm{H}_{3}$

c; $\mathrm{Ar}^{1}=$ thiophen-2-yl, $\mathrm{Ar}^{2}=3,4,5-\left(\mathrm{OCH}_{3}\right)_{3} \mathrm{C}_{6} \mathrm{H}_{2}$<smiles>COc1ccc(C=O)cc1OC</smiles>

6a<smiles>COc1cc(C=O)cc(OC)c1OC</smiles>

6b

Scheme 1: synthesis of pyrimidine and thiazolopyrimidine derivatives. 
Epoxide derivatives 2a-c were reacted with thiourea to furnish the thioxopyrimidinone derivatives $\mathbf{8 a - c}$, respectively. The IR spectra for compounds 8a-c showed absorption bands for the $\mathrm{NH}$ and the ${ }^{1} \mathrm{H}$ NMR spectra showed signals for pyrimidine $\mathrm{H}-4$ and H-6. The presence of such signals each as doublet in ${ }^{1} \mathrm{H}$ NMR data revealed that the pyrimidine moiety in such derivatives is alicyclic, with a twist boat conformation (Flefel et al., 2007). Pyrimidine derivative 8a-c were reacted with chloroacetic acid and formed compounds 9a-c (Scheme 2). The IR spectra of the latter compounds showed absorption band for carbonyl groups. The signals corresponding singlet signal of the $\mathrm{CH}_{2}$ appeared in the ${ }^{1} \mathrm{H}$ NMR spectra in addition to aryl and pyrimidine protons. The thioxopyrimidine derivatives $\mathbf{8 b}, \mathbf{c}$ were allowed to react with chloroacetic acid and 3,4-dimethoxybenzaldehyde or 3,4,5trimethoxybenzaldehyde and furnished the arylidine substituted thiazolo[3,2-a]pyrimidine derivatives $\mathbf{1 0 b}, \mathbf{c}$ respectively. The oxirane derivatives $\mathbf{2 a - c}$ were reacted with thiosemicarbazide to produce compounds 11a-c. Also, compounds 2a,c were reacted with urea in presence of potassium hydroxide to form compounds 12a,c. Epoxide derivative $\mathbf{2 a}$ was reacted with guanidine hydrochloride in potassium hydroxide to afford the tetrahydropyrimidin-2(1H)-imine compound 13a. Reaction of the unsaturated ketones $\mathbf{2 b , c}$ with carbon disulfide in potassium hydroxide to afforded 1,3-oxathiolane derivatives $\mathbf{1 4 b}, \mathbf{c}$. The ${ }^{1} \mathrm{H}$ NMR of 14b,c showed the doublet signals of the oxathiolane ring in addition to aromatic signals.<smiles>O=C1CSC2=NC([AlH2])C(=O)N12</smiles>

9a-c<smiles>O=C1C([AlH2])N=c2sc(=C[AlH2])c(=O)n2C1[AlH2]</smiles>

$10 \mathrm{~b}, \mathrm{c}$

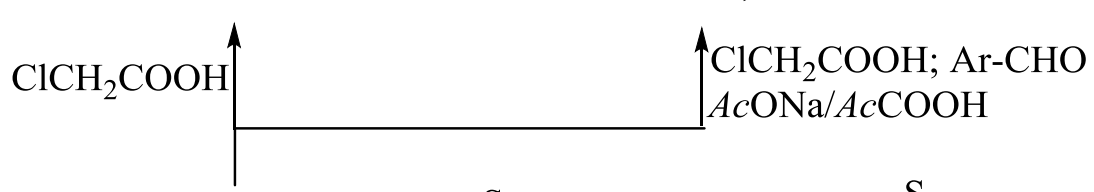

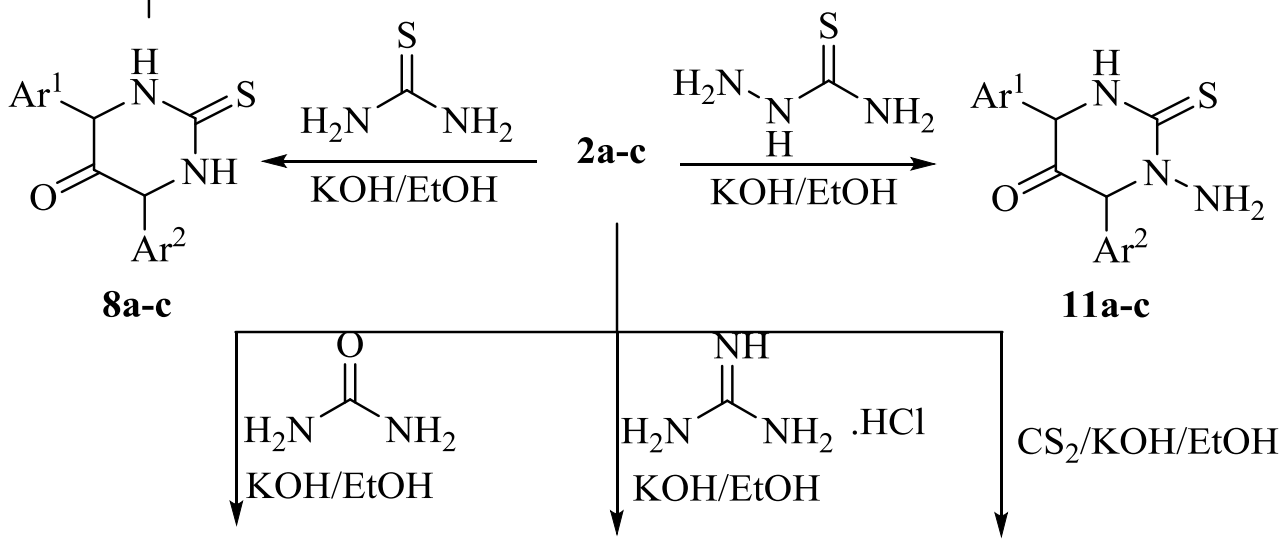<smiles>O=C1NC([Al])C(=O)C([Al])N1</smiles>

$12 \mathbf{a}, \mathbf{c}$<smiles>N=C1NC([Al])C(=O)C([Al])N1</smiles>

$13 \mathbf{a}$<smiles>O=C([AlH2])C1OC(=S)SC1[Al]</smiles>

$14 b, c$

a, $\mathrm{Ar}^{1}=$ thiophen-2-yl, $\mathrm{Ar}^{2}=-\mathrm{C}_{6} \mathrm{H}_{5}$

b, $\mathrm{Ar}^{1}=$ thiophen-2-yl, $\mathrm{Ar}^{2}=3,4-\left(\mathrm{OCH}_{3}\right)_{2} \mathrm{C}_{6} \mathrm{H}_{3}$

c, $\mathrm{Ar}^{1}=$ thiophen-2-yl, $\mathrm{Ar}^{2}=3,4,5-\left(\mathrm{OCH}_{3}\right)_{3} \mathrm{C}_{6} \mathrm{H}_{2}$

Scheme 2: Synthesis of amino-and iminopyrimidine and thiazolopyrimidine derivatives. 
When compound 8a was reacted with the acetylated glycopyranosyl bromide for glucose and xylose moieties, in basic medium, the corresponding acetylaed thioglycosides $\mathbf{1 5 a}, \mathbf{b}$ were afforded in good yields. The infra-red spectra of the glycosides 15a,b indicated the bands in the range $1742-1750 \mathrm{~cm}^{-1}$ for the acetyl carbonyl groups. The ${ }^{1} \mathrm{H}$ NMR spectra showed peaks assigned for the protons of the sugar moiety and carbonyl methyl protons. The sugar moiety attachment at the sulfur center rather than the nitrogen atom was also confirmed by the disappearance of a signal corresponding to the $\mathrm{C}=\mathrm{S}$ in the ${ }^{13} \mathrm{C}$ NMR spectrum of 15a. Deacetylation of the acetylated thioglycosides 15a,b in methanol saturated with ammonia produced the free hydroxy thioglycosides 16a,b (Scheme 3). The IR spectra of the deacetylated products $\mathbf{1 6 a}, \mathbf{b}$ showed absorption bands for the hydroxyl groups and also revealed the absence of the acetyl carbonyl bands. ${ }^{1} \mathrm{H}$ NMR spectra of compounds 16a,b showed signals corresponding to the hydroxyl protons.

$8 \mathbf{a}$

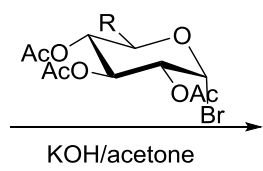

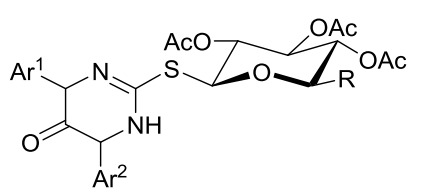

15a; $\mathrm{R}=\mathrm{CH}_{2} \mathrm{OAC}$ $15 b ; R=H$ $\downarrow \mathrm{NH}_{3} / \mathrm{CH}_{3} \mathrm{OH}$<smiles>[R]C1O[C@H](SC2=NC([Al])C(=O)C([Al])N2)C(O)C(O)C1O</smiles>

16a; $\mathrm{R}=\mathrm{CH}_{2} \mathrm{OH}$

$16 b ; R=H$

$$
\mathrm{Ar}^{1}=\text { thiophen-2-yl, } \mathrm{Ar}^{2}=\mathrm{C}_{6} \mathrm{H}_{5}
$$

Scheme 3: synthesis of pyrimidine thiolglycosides.

\section{Cytotoxic activity}

The cytotoxic activity of prepared compounds was investigated for their activity with respect to HepG-2, PC-3 and HCT-116 cell lines and such activity behavior has been estimated using MTT assay (Awad et al., 2014; Soliman et al., 2014).

The percentage of the intact cells was determined and compared to the control (table 1 and Fig. 2). The cytotoxicity of compounds under test towards the previously mentioned carcinoma cells were compared with that of Doxorubicin ${ }^{\circledR}$.

The afforded results indicated that the tested compounds exhibited dose-dependent behavior against the three cancer cell lines. From Table 1 we can deduce that, at $100 \mu \mathrm{g} / \mathrm{mL}$, two compounds (11b and 12a) showed good cytotoxic activities against HCT-116 carcinoma cells. Compounds 3a, 5c, 7c, 8a and 11a showed moderate activities and the remaining compounds displayed weak activities with respect to HCT-116 cells. In addition, in case of PC-3 cancer cells, the synthesized compounds; 7c and 12a showed high cytotoxic activity when compared with the reference drug. Furthermore, compound 8a showed also good cytotoxic activity against such cell line. Three compounds showed moderate activities (3a, 11a and 11b) and the rest of the compounds showed weak or no cytotoxic activities against this type of cell lines. Furthermore, all the compounds showed weak or no cytotoxic activities against HepG-2 liver cancer.

Table 1: Cytotoxic activity of compounds against cancer cell lines at $100 \mathrm{ppm}$.

\begin{tabular}{cccc}
\hline Compound & HCT-116 & PC-3 & HepG-2 \\
\hline $3 \mathrm{a}$ & 73.42 & 61.81 & 24.88 \\
$5 \mathrm{c}$ & 74.60 & 54.67 & 39.59 \\
$7 \mathrm{c}$ & 78.36 & 75.07 & 32.80 \\
$8 \mathrm{a}$ & 76.55 & 71.83 & 17.59 \\
$9 \mathrm{a}$ & 55.18 & 12.69 & 1.20 \\
$11 \mathrm{a}$ & 77.63 & 59.97 & 18.40 \\
$11 \mathrm{~b}$ & 81.99 & 68.79 & 20.44 \\
$12 \mathrm{a}$ & 85.84 & 75.93 & 18.36 \\
Doxorubicin & 92.45 & 72.21 & 88.14 \\
\hline
\end{tabular}

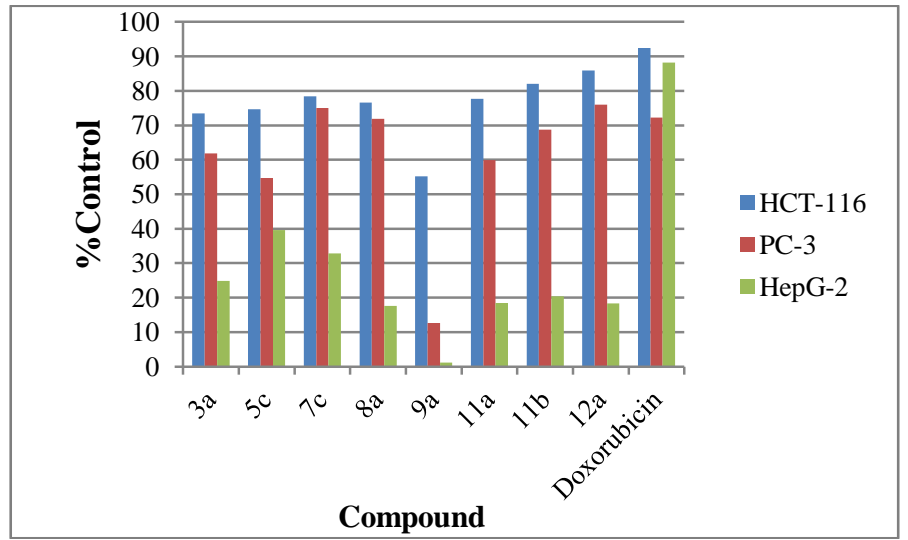

Fig. 2: Cytotoxic activity of compounds against cancer cell lines at $100 \mathrm{ppm}$.

Table 2: $\mathrm{IC}_{50}$ values for synthesized compounds against cancer cell lines.

\begin{tabular}{cccc}
\hline \multirow{2}{*}{ Compound } & HCT-116 & PC-3 & HepG-2 \\
\cline { 2 - 4 } & \multicolumn{3}{c}{ IC $_{\mathbf{5 0}}(\boldsymbol{\mu g} / \mathbf{m L})$} \\
\hline 3a & $68.1 \pm 3.6$ & $80.8 \pm 3.1$ & $200.8 \pm 4.8$ \\
$\mathbf{5 c}$ & $67.0 \pm 2.9$ & $91.4 \pm 3.2$ & $126.2 \pm 4.3$ \\
$\mathbf{7 c}$ & $63.8 \pm 3.2$ & $66.5 \pm 3.6$ & $152.4 \pm 3.2$ \\
$\mathbf{8 a}$ & $65.3 \pm 3.1$ & $69.6 \pm 2.1$ & $284.1 \pm 6.5$ \\
9a & $90.6 \pm 2.6$ & $393.9 \pm 4.5$ & $>1000$ \\
11a & $64.4 \pm 3.1$ & $83.3 \pm 2.5$ & $271.7 \pm 5.9$ \\
11b & $60.9 \pm 1.8$ & $72.6 \pm 3.2$ & $244.5 \pm 4.7$ \\
12a & $58.2 \pm 5.1$ & $65.8 \pm 2.8$ & $272.2 \pm 5.9$ \\
Doxorubicin & $73.50 \pm 2.9$ & $75.24 \pm 4.1$ & $67.9 \pm 3.2$ \\
\hline
\end{tabular}

By correlation of the afforded cytotoxic activity results with structural features of tested compounds it may be deduced that substitution of the thiopyrimidine nucleus at ring nitrogen $\left(N^{3}\right)$ with amino group resulted in an enhanced effects in case of incorporation of phenyl moiety as an aryl function at $C-6$ in the pyrimidine ring. Furthermore, substituted pyrimidine derivatives, with dimethoxyphenyl at $C-6$, revealed more activity against HCT116 and PC-3 cell lines than their analogues incorporating trimethoxyphenyl moiety. The free 1,3-unsubstituted pyrimidinedione derivatives showed relatively higher activity against HCT-116 and PC-3 cell lines than the corresponding 2- 
thioxo analogues. Obviously, the substituted diarylthiazolopyrimidine nucleus free of oxo-substitution at $C-6$ was clearly found to be more active than the corresponding 6-oxo analogue as the activity was lost in compound $\mathbf{1 0}$ in which $C-6$ was functionalized with oxo- group. Furthermore, substitution at the methylene carbon in the thiazolopyrimidine system with arylidine function, in absence of oxo- group at $C-6$, led to more active derivatives than their precursors with free $\mathrm{CH}_{2}$ group, especially in presence of trimethoxyphenyl moiety.

\section{CONCLUSION}

New substituted oxo- and thioxopyrimidine, thiazolopyrimidine and pyrimidine thioglycoside derivatives were synthesized and structurally characterized. The prepared compounds showed cytotoxic activity against HCT-116 and PC-3 cell lines revealing moderate to good activities. A number of compounds revealed good effectiveness especially on PC-3 cell line and could be believed as valuable templates for further investigations to get more potent agents.

\section{Financial support and sponsorship: Nil.}

Conflict of Interests: There are no conflicts of interest.

\section{REFERENCES}

Abdel Mohsen HT, Ragab FAF, Ramla MM, El Diwani HI. Novel benzimidazole-pyrimidine conjugates as potent antitumor agents. Eur J Med Chem 2010; 45: 2336-2344.

Alagarsamy V, Mecna S, Ramseshu KV, Solomon VR, Thirumvnugan K, Dhanabal K, Murugan M. Synthesis, analgesic, antiinflammatory, ulcerogenic index and antibacterial activities of novel 2methylthio-3-substituted-5,6,7,8-tetrahydrobenzo(b)thieno

$[2,3-$ d]pyrimidin-4(3H)-ones. Eur J Med Chem 2006; 41: 1293-1300.

Alagarsamy V, Pathak US, Revathi R. Anticancer, antibacterial and antifungal activities of soidc 2-substituted (1,3,4)thiadiazolothieno(2,3c)pyrimidin-5-(4H)-ones. Ind $\mathrm{J}$ Heterocycl Chem 2003; 12 : 335-338.

Amr AE-G, Maigali SS, Abdulla MM. Synthesis, and analgesic and antiparkinsonian activities of thiopyrimidine, pyrane, pyrazoline, and thiazolopyrimidine derivatives from 2-chloro-6-ethoxy-4-acetylpyridine. Monatsh. Chem 2008; 139: 1409-1415.

Awad HM, Abd-Alla HI, Mahmoud KH, El-Toumy SA. In vitro anti-nitrosative, antioxidant, and cytotoxicity activities of plant flavonoids: A comparative study. Med Chem Res 2014; 23: 3298-3307.

Brault L, Migianu E, Néguesque A, Battaglia E, Bagrel D, Kirsch G. New thiophene analogues of kenpaullone: synthesis and biological evaluation in breast cancer cells. Eur J Med Chem 2005; 40: 757-763.

Chou YM, Lai MC, Hwang TM, Ong CW. Synthesis and antitumor activity of bithienyl-pyrimidine derivatives with electrostatic binding side chains. Bioorg Med Chem Lett 1999; 9: 2643-2646.

Da EP, Falcao S, De Melo SJ, Srivastava RM, De MTJ, Catanho A, Nascimento, SCD. Synthesis and antiinflammatory activity of 4-amino -2-aryl-5-cyano-6-\{3- and 4-(N-phthalimidophenyl) $\}$ pyrimidines. Eur J Med Chem 2006; 41: 276-282.

El Ashry ESH, Kassem AA, Abdel-Hamid H, Louis FF, Khattab ShAN, Aouad, MR. Novel Regioselective Formation of S- and NHydroxyl-Alkyls of 5-(3-Chlorobenzo[b]Thien-2-yl)-3-Sulfanyl-4H-1,2,4Triazole and A Facile Synthesis of Triazolo-Thiazoles and ThiazoloTriazoles. Role of Catalyst and Microwave. Nucleosides Nucleotides Nucleic Acids 2007; 26: 437-451.
El-Sayed WA, Abbas HS, Abdel Magid RE, Magdziarz T. Synthesis, Antimicrobial Activity and Docking Studies of New 3(Pyrimidin-4-yl)-1H-indole Derivatives and Their Derived N- and Sglycoside Analogs. Med Chem Res 2016; 25: 339-355.

El-Sayed WA, Abdel-Rahman AA-H, Ramiz MMM. AntiHepatitis B Virus Activity of New $\mathrm{N}^{4}-\beta$-D-Glycoside pyrazolo[3,4d]pyrimidine derivatives $Z$ Naturforsch 2009; 64c: 323-328.

El-Sayed WA, El-Sofany WI, Hussein HA, Fathi NM. Synthesis and Anticancer Activity of New [(Indolyl)pyrazolyl]-1,3,4-oxadiazole Thioglycosides and Acyclic Nucleoside Analogs. Nucleosides, Nucleotides and Nucleic Acids 2017; 36: 474-495.

El-Sayed WA, Fathi NM, Gad WA, El-Ashry ESH. Synthesis and Antiviral Evaluation of Some 5-N-Arylaminomethyl-2glycosylsulphanyl-1,3,4-oxadiazoles and Their Analogs against Hepatitis A and Herpes Simplex Viruses. J Carbohydr Chem 2008; 27: 357-372.

El-Sayed WA, Rashad AE, Awad SM, Ali MM. Synthesis and in vitro Antitumor Activity of Some New Substituted Thiopyrimidines Through Radical Balance Regulation. Nucleosides, Nucleotides and Nucleic Acids 2009; 28: 261-274.

El-Sayed WA, Abdel-Rahman AA-H. Copper-catalyzed Synthesis and Antimicrobial Activity of Disubstituted 1,2,3-Triazoles Starting from 1-Propargyluracils and Ethyl (4-azido-1,2,3trihydroxybutyl)furan-3-carboxylate. Z Naturforsch 2010; 65B: 57-66.

Fahmy HTY, Rostom SAF, Saudi MN, Zjawiony JK, Robins DJ. Synthesis and in vitro evaluation of the anticancer activity of novel fluorinated thiazolo[4,5-d]pyrimidines. Arch Pharm Pharm Med Chem 2003; 3: 1-10.

Flefel EE, Salama MA, El-Shahat M, El-Hashash MA, ElFarargy AF. A novel synthesis of some new pyrimidine and thiazolopyrimidine derivatives for anticancer evaluation. Phosph. Sulfur Silic Rel Elem 2007; 182: 1739-1756.

Flefel EM, El-Sayed WA, Mohamed AM, El-Sofany WI, Awad HM. Synthesis and Anticancer Activity of New 1-Thia-4azaspiro[4.5]decane, Their Derived Thiazolopyrimidine and 1,3,4Thiadiazole Thioglycosides Molecules 2017; 22: 170-182.

Geist JG, Lauw S, Illarinova V, Fischer M, Gwawert $\mathrm{T}$, Rohdich F, Eisenreich W, Kaiser J, Groll M, Scheurer C et al. Thiazolopyrimidine inhibitors of 2-methylerythritol 2,4-cyclodiphosphate synthase (IspF) from Mycobacterium tuberculosis and Plasmodium falciparum. ChemMedChem 2010; 5: 1092-1101.

Gholap AR, Toti, KS, Shirazi F, Deshpande MV, Srinivasan $\mathrm{KV}$. Efficient synthesis of antifungal pyrimidines via palladium catalyzed Suzuki/Sonogashira cross-coupling reaction from Biginelli 3,4dihydropyrimidin-2(1H)-ones. Tetrahedron 2008; 64: 10214-10223.

Gillespie RJ, Bamford, SJ, Clay A, Gaur S, Haymes T, Jackson PS, Jordan AM, Klenke B, Leonardi S, Liu J, Mansell HL, Ng S, Saadi M, Simmonite H, Stratton GC, Todd RS, Williamson DS, Yule IA. Antagonists of the human A2A receptor. Part 6: Further optimization of pyrimidine-4-carboxamides. Bioorg Med Chem 2009; 17: 6590-6605.

Greiner-Bechert L, Otto H-H. 1,4-Pentandien-3-ones, XXXII: Reaction of 2-acetylthiophene and 2-acetylfuran with malononitrile and aldehydes, and synthesis and properties of phenylenebis[(thienyl/furyl)nicotinonitrile] derivatives. Arch Pharm 1991; 324: 563-572.

Haiba ME, Fathalla OA, Zeid IF, Soliman AM, Abd El-Moez SI, El-serwy, WS. Synthesis and evaluation of some novel tetrahydropyrimidine derivatives as antimicrobial and cytotoxic agents. Res Chem Intermed 2013; 39: 3763-3774.

Haouz A, Vanheusden V, Munier-Lechman H, Froeyen M, Herdewijn P, Van Calenbergh S, Delarue M. Enzymatic and Structural Analysis of Inhibitors Designed against Mycobacterium tuberculosis Thymidylate Kinase: New insights into the Phosphoryl Transfer Mechanism J Biol Chem 2003; 278: 4963-4971.

Jordheim LP, Durantel D, Zoulim F, Dumontet C. Advances in the development of nucleoside and nucleotide analogues for cancer and viral diseases. Nat Rev Drug Disc 2013; 12: 447-64. 
Kolb S, Mondésert O, Goddard ML, Jullien D, Villoutreix BO, Ducommun B, Garbay C, Braud E. Development of novel thiazolopyrimidines as CDC25B phosphatase inhibitors. ChemMedChem 2009; 4: 633-648.

Kumar G, Aggarwal H, Gupta R. Cobalt Complexes Appended with para- and meta-Arylcarboxylic Acids: Influence of Cation, Solvent, and Symmetry on Hydrogen-Bonded Assemblies. Cryst Growth Des 2013; 13: 74-90.

MacCoss M, Robins MJ in: D.E.V. Wilman (Ed.), The Chemistry of Antitumor Agents, Chapman and Hall, New York, (1990) pp. 261-298.

Maurinsh Y, Schraml J, De Winter H, Blaton N, Peeters O, Lescrinier E, Rozenski J, Van Aerschot A, De Clercq E, Busson R, Herdewijn P. Synthesis and Conformational Study of 3-Hydroxy-4(Hydroxymethyl)-1-Cyclohexanyl Purines and Pyrimidines. J Org Chem 1997; 62: 2861-2871.

Ostrowski T, Wroblowski B, Busson R, Rozenski J, De Clercq E, Bennet MS, Champness JN Summers WC, Sanderson MR, Herdewijn P. 5-Substituted Pyrimidines with a 1,5-Anhydro-2,3-dideoxy-d-arabinohexitol Moiety at N-1: Synthesis, Antiviral Activity, Conformational Analysis, and Interaction with Viral Thymidine Kinase. J Med Chem 1998; 41: 4343-4353.

Qiang $\mathrm{H}, \mathrm{Wu} \mathrm{J}-\mathrm{W}, \mathrm{Xu} \mathrm{H}-\mathrm{J}$. Biomimetic hydrogenation: a reusable NADH co-enzyme model for hydrogenation of $\alpha, \beta$-epoxy ketones and 1,2-diketones. Tetrahed Lett 2013; 54: 3877-3881.

Ramesh B, Rao BS. Synthesis, Spectral Studies and AntiInflammatory Activity of 2-Acetyl Thiophene. E-J Chem 2010; 7: 433436.

Ramez MM, El-Sayed WA, Tantawy AA, Abdel Rahman AAH. Antimicrobial Activity of New 4,6-Disubstituted Pyrimidine, Pyrazoline, and Pyran Derivatives. Arch Pharm Res 2010; 33: 647-654.

Rashad AE, Shamroukh AH, Abdel-Megeid RE, El-Sayed WA. Synthesis, reactions and antimicrobial evaluation of some polycondensed thieno-pyrimidine derivatives. Synth Commun 2010; 40: 1149-1160.

Said M, Abouzid K, Mouneer A, Ahmedy A, Osman A-M. Synthesis and biological evaluation of new thiazolopyrimidines. Arch Pharm Res 2004; 27: 471-477.

Siddiqui AA, Rajesh R, Islam MU, Alagarsamy V, De Clercq E. Synthesis, Antiviral, Antituberculostic, and Antibacterial Activities of Some Novel, 4-(4-substituted phenyl)-6-(4-nitrophenyl)-2-(substituted imino)pyrimidines ). Arch Pharm 2007; 340: 95-102.

Singh P, Paul K. Anti-cancer activities of 5-acyl-6-[2hydroxy/benzyloxy-3-(amino)-propylamino]-1,3-dialkyl-1H-pyrimidin2,4-diones. Bioorg Med Chem 2006; 14: 8622-8625.
Soliman HA, Yousif MNM, Said MM, Hassan NA, Ali MM, Awad HM. Synthesis of novel 1,6-naphthyridines, pyrano[3,2-c]pyridines and pyrido[4,3-d]pyrimidines derived from 2,2,6,6-tetramethylpiperidin-4one for in vitro anticancer and antioxidant evaluation. Der Pharma Chemica 2014; 6: 394-410.

Spanou C, Manta S, Komiotis D, Dervishi A, Kouretas D. Antioxidant Activity of a Series of Fluorinated Pyrano-nucleoside Analogues of N4-benzoyl Cytosine and $\mathrm{N}^{6}$-benzoyl Adenine. Int J Mol Sci 2007; 8: 695-704.

Srour AM, Ismail AA, El-Kosy SM, Zeid IF. Antiviral and Antischistosomal Evaluation of Newly Synthesized Thioglycosides and their Acyclic Analogues. Z Naturforsch 2009; 64c: 483-489.

Summa V, Petrocchi A, Bonelli F, Crescenzi B, Donghi M, Ferrara M, Fiore F, Gardelli C, Paz OG, Hazuda DJ, Jones P, Kinzel O, Laufer R, Monteagudo E, Muraglia E, Nizi E, Orvieto F, Pace P, Pescatore G, Scarpelli R, Stillmock K, Witmer MV, Rowley M. Discovery of Raltegravir, a Potent, Selective Orally Bioavailable HIV-Integrase Inhibitor for the Treatment of HIV-AIDS Infection. J Med Chem 2008; 51 : 5843-5855.

Vastmans K, Froeyen M, Kerremans L, Pochet S, Herdewijn P. Reverse transcriptase incorporation of 1,5-anhydrohexitol nucleotides. Nucleic Acids Res 2001; 29: 3154-3163.

Zhi H, Chen L, Zhang L, Liu S, Wan DCC, Lin H, Hu C. Design, synthesis, and biological evaluation of $5 \mathrm{H}$-thiazolo[3,2a]pyrimidine derivatives as a new type of acetylcholinesterase inhibitors. ARKIVOC 2008; xiii: 266-277.

How to cite this article:

Yousif MNM, El-Sayed WA, Abbas HS, Awad HM, Yousif NM. Anticancer Activity of New Substituted Pyrimidines, Their Thioglycosides and Thiazolopyrimidine Derivatives. J App Pharm Sci, 2017; 7 (11): 021-032. 